\title{
EXERGAME USE AS A GATEWAY TO THE ADOPTION OF AND ADHERENCE TO SPORT-SPECIFIC AND GENERAL PHYSICAL ACTIVITY
}

\author{
by \\ COLIN JENNEY \\ Presented to the Faculty of the Graduate School of \\ The University of Texas at Arlington in Partial Fulfillment \\ of the Requirements \\ for the Degree of \\ MASTER OF SCIENCE IN PSYCHOLOGY
} THE UNIVERSITY OF TEXAS AT ARLINGTON August 2012 
Copyright ( by Colin Jenney 2012

All Rights Reserved 


\section{ACKNOWLEDGEMENTS}

I would like to first thank my committee members including Angela Liegey Dougall, PhD, Linda Perrotti, PhD, Pablo Mora, PhD, and Judy Wilson, PhD. I greatly appreciate your time and advice for the enrichment of this project. I would like to give special thanks to Judy Wilson, PhD, Becky Garner, Brad Heddins, MS and Abu Yilla, PhD. These individuals and the staff of the Kinesiology Department provided me with many resources that made this research possible. Furthermore, I would also like to give special thanks to the MAC staff, especially Durl Rather and Blair Schuyler, for their assistance in providing me with the facilities and resources to function.

Acknowledgement must also be given to my research assistants John Havens, Alyssa Mitte, Padraic Murphy and Garrett Gatchel for all their assistance during this project. I also want to recognize my fellow graduate student and friend Jeff Swanson, MA for his years of support that directly contributed to this research. Additionally, I want to thank the most important person in my life, my spouse Kathy Jenney, for her undying support and love that gave me the strength to put in countless late nights and early mornings in the name of research.

Finally, I wish to give extra special thanks to my wonderful mentor Angela Liegey Dougall. Your years of advice, support and guidance for this project as well as for countless others will never be forgotten.

July 13,2012 


\author{
ABSTRACT \\ EXERGAME USE AS A GATEWAY TO THE ADOPTION OF AND \\ ADHERENCE TO SPORT-SPECIFIC AND \\ GENERAL PHYSICAL ACTIVITY
}

Colin Jenney, M.S.

The University of Texas at Arlington, 2012

Supervising Professor: Angela Liegey Dougall

Despite increasing the amount of information regarding the need for regular physical activity, rates of inactivity have not seen a corresponding decrease. The aim of the current study was to determine whether exergame use would serve as a gateway to the sport of racquetball, thereby providing a feasible and innovative way to influence behavior change regarding physical activity. The current study randomly assigned participants into one of three conditions for racquetball training: a no-exposure control group, a racquetball videogame (exergame) group, and a traditional training group. College aged adults $(N=103)$, who reported not being regular exercisers at recruitment and who had no prior experience with racquetball, followed the training method outlined by their group assignment for Weeks $0-4$, after which all groups participated in the same, traditional racquetball training for Weeks $5-8$. It was expected that the exergame group would have greater levels of skills test performance, general physical activity, fitness center use, levels of accelerometer activity, enjoyment, self-efficacy, and intention for physical activity. Concurrently, the exergame group was also expected to experience greater than or equal scores for these measures when compared to the traditional training group; supporting 
exergame use as either a superior or equivalent training method compared to the traditional training method. Furthermore, enjoyment and self-efficacy were expected to mediate the relationship between condition, and the physical and cognitive measures of physical activity. ANCOVA models revealed that the exergame group had greater skills test performance than the control group at T3 (for only males) and that no differences were found between the exergame and traditional training groups for the variables of enjoyment, skills test performance, racquetball self-efficacy, and activity (Weeks $5-8$ ). However, greater enjoyment, self-efficacy measures, accelerometer activity, future intention, general physical activity and fitness center use were not seen for the exergame group above all other groups and the traditional training group was found to exhibit greater activity values on most outcomes. Analyses also discovered increases across time for all outcome variables regardless of group, with the exception of exercise self-efficacy. Due to these findings, the analyses testing exercise self-efficacy and enjoyment as mediators of group effects on the other outcomes could not be assessed. These results provide partial support for the novel use of exergames as a gateway to physical activity, and provide guidelines for future research to consider including gender, background exercise experience, and specific activity oriented differences. 
TABLE OF CONTENTS

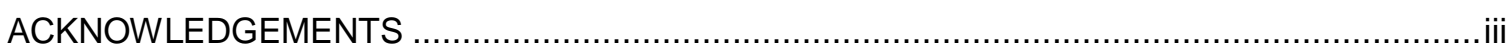

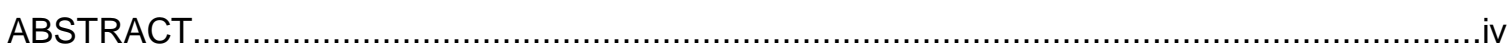

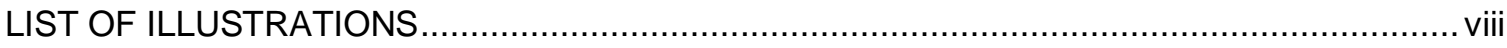

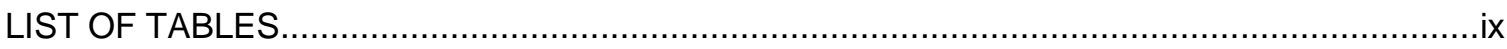

$\begin{array}{lll}\text { Chapter Page } & \text { Pag }\end{array}$

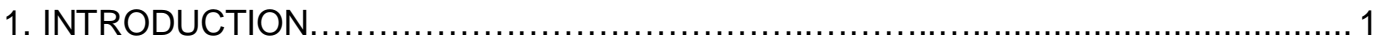

1.1 Exergame Research and Specific Populations..................................... 2

1.2 Theoretical Background and Construction of Concepts ........................... 5

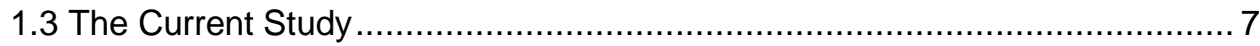

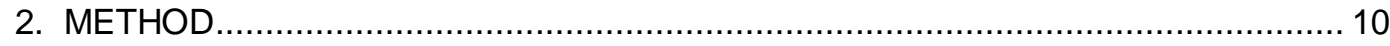

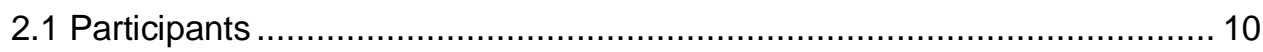

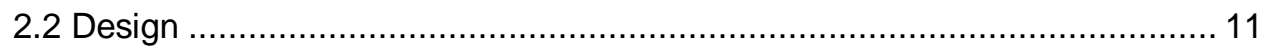

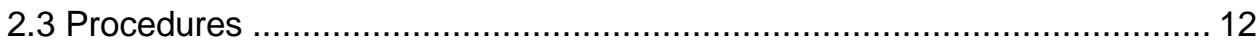

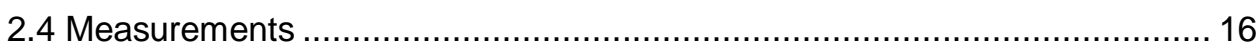

2.4.1 Demographics and Health................................................. 17

2.4.2 Previous Activity Experience …............................................ 17

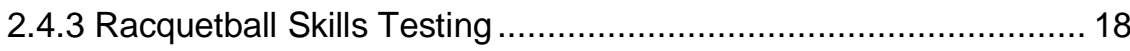

2.4.4 Activity Center Use ............................................................ 18

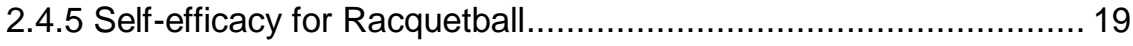

2.4.6 Enjoyment of Racquetball .............................................. 19

2.4.7 Future Intention for Physical Activity...................................... 20

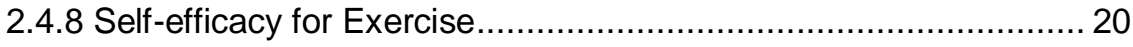

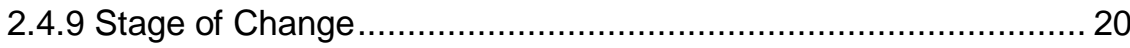


2.4.10 Racquetball Participation.................................................. 21

2.4.11 Activity Levels........................................................... 21

2.4.12 Participant Feedback ........................................................ 22

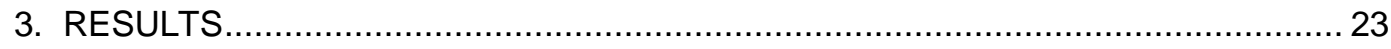

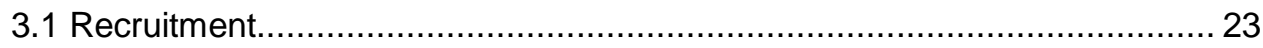

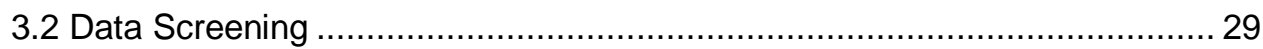

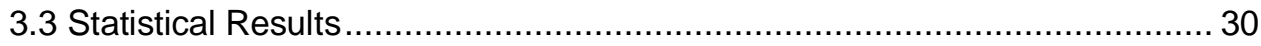

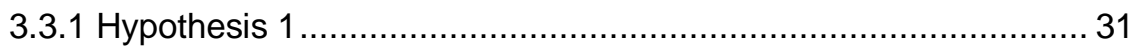

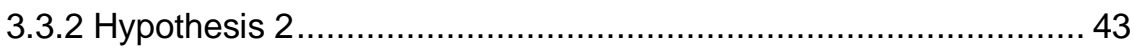

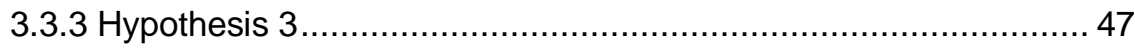

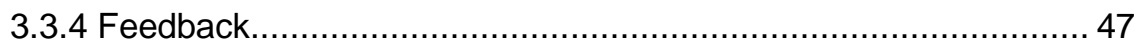

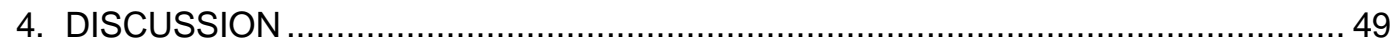

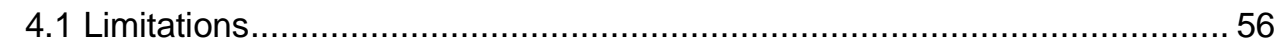

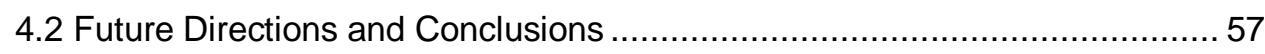

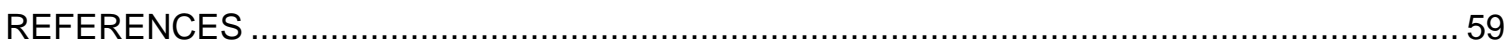

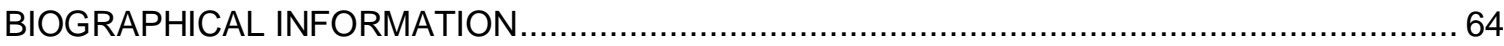




\section{LIST OF ILLUSTRATIONS}

$\begin{array}{ll}\text { Figure } & \text { Page }\end{array}$

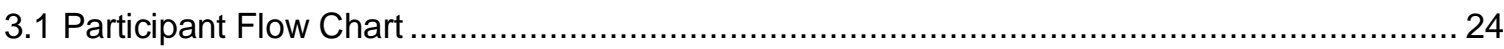

3.2 Skills Test Performance for the Significant Interaction of Time

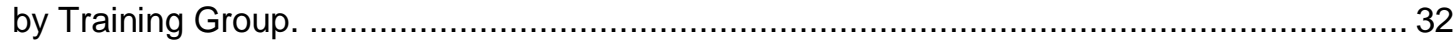

3.3 Skills Test Performance for the Significant Interaction of Time

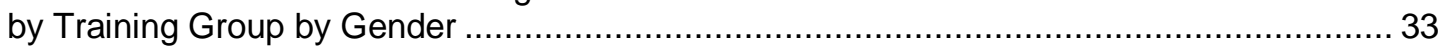

3.4 Activity (accelerometer counts) for the Significant Interaction

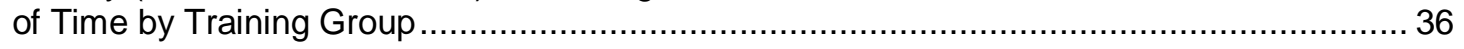

3.5 Activity (accelerometer counts) for the Significant Interaction

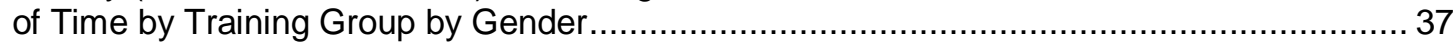

3.6 Racquetball Self-efficacy for the Significant Interaction of

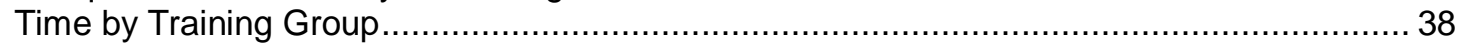

3.7 Racquetball Self-efficacy for the Significant Interaction of

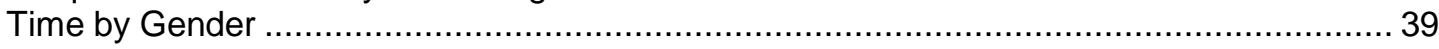

3.8 Enjoyment for the Significant Interaction of Time by Training Group...

3.8 Enjoyment for the Significant Interaction of Time by Training Group by Gender 


\section{LIST OF TABLES}

Table

Page

3.1 Demographic Characteristics by Training Group

3.2 Training Group Means and Standard Errors for Hypothesis 1 Outcomes at Week 0 - Baseline (T1),

After Week 4 - Transition (T2), and After Week 8

- Completion of Training (T3).

3.3 Training Group Means and Standard Errors for

Activity for Hypothesis 1 at Weeks 1 - 8

3.4 Training Group Means and Standard Errors for Hypothesis 2 Outcomes at Week 0 - Baseline (T1), After Week 4 - Transition (T2), and After Week 8

- Completion of Training (T3). 45

3.5 Descriptive Statistics for Study Feedback Variables. 


\section{CHAPTER 1}

\section{INTRODUCTION}

As a multitude of recent findings support the failure of Americans to participate in adequate levels of physical activity, innovative methods are becoming increasingly necessary in order to reverse this trend. The use of exergames to positively influence physical activity behaviors has started to be used to increase levels of general physical activity, enjoyment, and decrease adversity to exercise (Jarvis, 2010, Murphy, Carson, Neal, Baylis, Donley, \& Yeater, 2009, \& Kepka, 2011). Specifically, recent research has suggested exergames increased willingness to participate in sports within adolescents. These findings suggest that exergames can be a gateway to the adoption of a specific-sport as well as general physical activities (Murphy et al., 2009 \& Kepka, 2011). It was the primary objective of this study to determine if exergames could be used as a gateway to adopting a particular physical activity (a specific sport) or general physical activity behaviors, how this method compares to a traditional training method, if exergames could increase future intention for physical activity, if self-efficacy for exercise and for a novel sport would be increased by exergame use, if enjoyment and selfefficacy act as mediators of the intervention, and whether or not exergame training could potentially be used as an intervention for increasing general physical activity among young adults.

As American organizations continue to report on the desperate need for individuals to engage in regular physical activity, it is often emphasized that health problems and lower quality of life have been unarguably linked to the lack of physical activity. It has been estimated that 250,000 American deaths every year can be attributed to the lack of regular exercise (US DHHS, 1996). A more recent report from the Center for Disease Control (CDC) stated that in 2007 , only $48.8 \%$ of Americans were reporting participation in the recommended amount of 
physical activity (CDC, 2010). Other recent reports have put the issue into a dire perspective by finding that physical inactivity accounted for greater deaths than did smoking (Lam, Ho, Hedley, Mak, \& Leung, 2004). Due to the lack of activity, information regarding exercise guidelines and thousands of resources such as websites, programs, and newsletters have been disseminated to Americans in an attempt to remedy this problem. However, in order to increase general levels of physical activity, these attempts must be supplemented with other methods to enhance their success.

Within the past two decades, physical activity inducing video games (exergames) have become a significant part of the market in the entertainment industry. These games differ from traditional sedentary video games by requiring that the player participate in the game through some sort of limited physical activity (e.g., dancing on a pad, moving a remote control around, or various movements of the body while standing). These data suggest that exergames could be used as "gateways" for modifying behaviors from those that are typically sedentary, to those that incorporate greater degrees of physical activity. The current project extended this idea to adoption of a specific, novel sport with a college population which is at high risk for sedentary behavior and weight gain. Given that exergames commonly simulate real world sports, there is a prospect that coordinated use of a particular sport exergame could help the player to become familiar with the rules, activities, and benefits of that sport, thereby allowing an easier transition into the actual sport. Despite the potential existence of this phenomenon, it has yet to be examined empirically.

\subsection{Exergame Research and Specific Populations}

Recent research has documented increases in physical activity and health benefits following interventions using exergames in samples consisting mostly of older adults and adolescents. Select studies have shown that exergames can increase general physical activity, balance, mobility, and range of motion when used in specific cases with the elderly (Jarvis, 2010). This exergame model has also been shown to be effective for slowing typical weight 
gain, increasing general physical activity and decreasing adversity to physical activity in adolescents (Murphy et al., 2009). Most interesting, this latter study showed that exergames produced marked increases in the willingness of adolescents to participate in sports according to self-report and parental-report measures. Despite the growing interest in this area, research has yet to be conducted with a sample of college students who are also vulnerable to decreasing levels of physical activity.

The lack of progress in increasing activity levels suggests that while providing more information and resources to sedentary individuals can slightly improve physical activity patterns, it does not alleviate this situation alone. Levels of physical activity can be changed through the implementation of behavioral interventions. While past interventions have been implemented (Project GRAD, Active Recreation Tertiary Education Campuses), the results of these studies were mixed in regard to what influential factors might deter or promote physical activity in a college-age population (Stone, McKenzie, Welk, \& Booth, 1998). This age group, specifically, are considered to be at a pivotal age for engaging in behaviors to promote and maintain physical activity. Unfortunately, these young adults also display steep decreases in physical activity after high school (ages $18-24$ yrs) when physical education courses are no longer mandatory. This lack of physical activity has been suggested to have a significant impact on morbidity and mortality rates in the United States (Grubbs \& Carter, 2002). For those in college, adjusting to a lifestyle that requires the student to keep a strict schedule between attending class, studying, extracurricular activities, and managing aspects of life outside of academia can be difficult. This routine has been known to exacerbate decreasing levels of physical activity, especially as a lack of time has been noted as one of the five most reported exercise barriers among this population (Dougall, Swanson, Grimm, Jenney \& Frame, 2011); Grubbs \& Carter, 2002). Therefore, the importance of intervening during this critical period is great. However, intervention strategies used must be designed to accommodate time 
constraints and be easily accessible on campus, as many college students live nearby (Dougall, et al., 2011).

Analysis of past literature in exergame research reveals that exergames may work by decreasing exercise barriers. Common barriers to exercise include lacking time, social embarrassment and intimidation, and perceiving exercise as difficult and adverse (Dougall, et al., 2011; Grubbs, \& Carter, 2002). Due to the nature of the gaming platforms, exergames have the potential to remove many exercise barriers by allowing the activity to be used at home, being relatively low in cost, having optional group-play games, and being enjoyable. Removing these barriers improves the likelihood of frequent and beneficial use of this medium and can concurrently improve cognitive perceptions of physical activity. Surveys of exercise barriers have also shown the commonly negative attitudes that participants hold for physical activity (Dougall, et al., 2011; Grubbs, \& Carter, 2002). By introducing or continuing physical activity through a medium that is generally perceived as fun, it is logical to expect an association to be established between the positive attitudes held for video games and engagement in physical activity.

In addition to physical health benefits, those who have participated in past exergame studies have also reported their experiences as highly enjoyable (Sell, Lillie, \& Taylor, 2008). Although this may seem like a trivial factor when lives are at stake, it has been shown to be an extremely useful tool to enhance the efficacy and intensity of a workout while increasing adherence rates. Past research has found positive associations between exercise intensity and enjoyment, as well as for adherence rates and enjoyment (Hagberg, Lindahl, Nyberg, \& Hellénius, 2009). Studies such as this suggested that enjoyment was an important mediator of exercise. Although exercise adoption is a crucial step in increasing general levels of physical activity, program initiation can be rendered useless without the participant's adherence. It has been reported that $50 \%$ of those who initiate an exercise program drop out inside of 6 months 
(Dishman, 1994). Given these concerns, the current study sought to increase enjoyment of physical activity through use of exergames, thereby increasing adherence rates.

Past exergame research has also suggested that most of the games for one of the major platforms (Nintendo Wii) fall within the realm of low, or light, intensity exercise. When measured for METs, all but a select few games were shown to produce energy expenditure in the range of low intensity (Miyachi, Yamamoto, Ohkawara, \& Tanaka, 2010). While past literature has promoted this finding as a downfall for the future of exergame use as a medium of physical activity, it can correspondingly be viewed as a merit for clinical exercise interventions. Current exercise recommendations from the American College of Sports Medicine suggested that introductory exercise regimens use a method which is safe, gradual and allows for progression (Haskell et al., 2007). Implementation of an exercise intervention using exergames would be able to use the light intensity physical activity as an appropriate introduction, while still allowing for progression.

\subsection{Theoretical Background and Construction of Concepts}

Exercise interventions have often been guided by various theoretical constructs in order to enhance their effects. Previous studies on designing exercise interventions have found support for using Stages of Change from the Transtheoretical Model introduced by Prochaska, DiClemente, \& Norcross (1992)(Marcus et al., 1992 \& Wallace, Buckworth, Kirby \& Sherman, 2000). The Stages of Change model theorized that people progress through stages on their way to changing problem behaviors, rather than making a single large step. This model stated that people move forward and backward through these stages, which included pre-contemplation (no intention to change behavior in the foreseeable future), contemplation (aware that a problem exists and thinking about overcoming it, but with no commitment yet), preparation (intending to take action in the next month, but have not been successful in the past year), action (modifications are made to behavior, experiences, or environment in order to overcome 
problems), and maintenance stages (work is put forth to prevent relapse and to consolidate gains attained during action stage).

While this model has aided in the development of physical activity interventions, it has not accounted for additional factors that predicted exercise behavior change, such as selfefficacy (belief in one's ability to succeed in a specific situation) and motivation. Previous research has used constituents of the Theory of Planned Behavior (Ajzen, 1985) to further explain movement through the Stages of Change as they pertained to health behavior change (Courneya, \& Bobick, 2000; Hausenblas, Carron, \& Mack, 1997). The Theory of Planned Behavior suggested that intentions for a behavior were central determinants for actually performing that behavior, influencing one's motivation. The concept of self-efficacy was particularly salient to the cognitive changes expected to occur with sport-specific exergame use. Self-efficacy has been described as operating on both motivation and action, where experience with the specific activity in question can cause positive shifts in these constructs (Bandura, 1998). While past research has not specifically focused on self-efficacy in relation to exergame use, the simulated experience gained during play was expected to increase self-efficacy in a manner similar to actual experience.

However, it must be noted that critics of the Theory of Planned Behavior have suggested that it best predicts intentions of behavior, rather than behavior itself (Norman \& Conner, 2005). Previous meta-analyses have reported significant associations between intention and behavior pertaining to exercise behaviors (Hagger, Chatzisarantis, \& Biddle, 2002; Norman \& Conner, 2005). However, the strength of these relationships varied greatly, suggesting that research needed to be conducted to assess the conditions in which intentions are more or less likely to predict behavior. Additionally, it has been reported that when past physical activity was accounted for, the influences of attitude and self-efficacy on the intentionbehavior relationship were greatly reduced (Hagger, Chatzisarantis, \& Biddle, 2002). The current study aimed to discover additional factors that influenced the intention-behavior 
relationships and/or successfully predicted exercise intention and behavior in a sample that was both sedentary and understudied in this area of research.

Previous literature concerning exercise behaviors has effectively shown that the concepts of self-efficacy and intention were significant predictors of physical activity (Wallace, Buckworth, Kirby \& Sherman, 2000; Hill, Abraham, \& Wright, 2007). The current study was designed to measure factors from the Theory of Planned Behavior including intention and selfefficacy and how they interact with individuals' movement through the stages of change. Use of this combined model helps to supplement the research on the efficacy of these models and will provide additional data on relationships between individual factors within these models.

\subsection{The Current Study}

The current study aimed to use a novel approach to changing exercise behavior by using a typically sedentary behavior, playing video games, to introduce exercise behaviors. An experimental design examined three different conditions of introductory training (None, Exergame, and Traditional Training) over an eight week period. This research examined exergames as a gateway to physical activity among college students who are not regular exercisers. The goals of this study were to decrease adversity to exercise, increase enjoyment, and introduce regular activity through introductory, low-intensity exercise. Aims of this research sought to demonstrate that playing a specific sport in an exergame will serve as a gateway to playing that actual sport in the real world, that a training program for this specific sport introduced through an exergame could be comparable or superior to traditional training methods, and that exergame use would increase future intention to exercise. Successful attainment of these aims would indicate that an intervention designed around these methods would aid sedentary young adults in the adoption and adherence to a regular exercise program, thus improving health outcomes. This method would reduce the aversion to physical activity that is common among those being introduced to exercise and should increase the enjoyment, selfefficacy, and future intention for performing exercise, all while allowing for a degree of 
progression. This prospective, randomized intervention has the potential to increase adoption and adherence by removing exercise barriers such as intimidation, embarrassment, issues with gym memberships, and low self-efficacy for general physical activity and specific sports.

Past literature entailing the ability of exergames to decrease exercise barriers and increase exercise enjoyment has supported the use of exergames as a viable introductory means of physical activity. Higher levels of enjoyment have also been shown to increase exercise intensity and commitment to exercise regimens thereby providing support for a possible mechanism by which exergames may increase physically active behaviors. Thus, exergame training should provide ample enjoyment with marked increases in self-efficacy for the sport being simulated. Based on these expectations, it was hypothesized that participants initially introduced to an exergame would report greater or equal performance on skills tests, activity (as measured by an accelerometer), and self-efficacy for the specific sport (racquetball) than those in the traditional training group, greater performance altogether than those in the control group, and greater enjoyment than all other groups, across all time points (Hypothesis 1).

Previous interventions in college-aged samples have found that disseminating information about exercise facilities may result in increases in facility use by making the individuals aware of the resources available to them. These previous findings, combined with the predicted increases in skill (due to training) and self-efficacy for exercise (due to experience), should predict similar findings for the current study. However, those in the exergame condition, who were expected to experience greater enjoyment, should correspondingly experience greater commitment to the activity than the other conditions. Therefore, it was expected that participants in the exergame condition would report greater levels of general physical activity, future intention for general physical activity, and greater fitness center use than all other groups, from the beginning to the end of the semester (Hypothesis 2). 
Enjoyment of physical activity has also been shown to mediate the effects of interventions on physical activity measures. These findings, paired with learning a physical activity through a typically fun and potentially familiar medium, were expected to also support exergame use. Thus, it was predicted that enjoyment and self-efficacy at the second assessment (between weeks 4 and 5) would mediate the relationship between condition and the outcome measures at the third assessment (after week 8), specifically skills test performance, racquetball self-efficacy, activity (as measured by an accelerometer), future intention for physical activity, activity center use, and actual physical activity (Hypothesis 3).

Collectively, these hypotheses were expected to demonstrate that exergame use could be a viable gateway to a particular physical activity by showing that the exergame group would have greater levels of the physical and cognitive physical activity measures than the control group; thus supporting the use of exergames as being an effective intervention. Concurrently, the exergame group was also predicted to experience greater than or equal scores for these measures when compared to the traditional training group; supporting exergame use as a superior or equivalent training method compared to the traditional training method. Additionally, the relationship between condition and the physical and cognitive measures of physical activity would be shown to be mediated by enjoyment and self-efficacy. 


\section{CHAPTER 2}

\section{METHOD}

\subsection{Participants}

One hundred and three participants who were not classified as regular exercisers were recruited through the Psychology Human Subjects Participant Pool as well from three professorapproved classes from within the Department of Kinesiology. Minimum sample size needed $(\mathrm{n}=98)$ was determined through an apriori power analysis for an analysis of variance using a medium effect size,$f=.25$, alpha of .05 , and power of .80 . Demographic characteristics of the participants closely matched those seen in previous studies using a similar participant population (Dougall, et al., 2011). The sample $(n=103)$ was comprised of 69 females $(68 \%)$ and 33 males (32\%), with a single missing case. The mean age of participants was $21.43 \pm 4.7$ years, with the majority reporting marital status as being single (87.3\%) and were not employed at the time (50.5\%). For ethnicity, 34\% reported being Hispanic, while $66 \%$ did not. Racial group representation was reported as $39 \%$ white, $24 \%$ black, $16 \%$ Asian, $1.9 \%$ American Indian/Alaskan Native, and $19 \%$ as other. The mean family income level for participants was reported as ranging from $\$ 30,001$ to $\$ 40,000$ yearly. It was also reported that the mean number of days in which participants' engaged in at least 20 minutes of vigorous physical activity per week was 1.72 . The study was placed on the Psychology SONA system to allow students to volunteer for the study, if they had already met prescreen criteria and if they chose to participate. Participants received 6 credits towards their required hours if they completed all phases of the study. However, partial credit was given to those who only completed the long online surveys and attended the orientation. Participants received 3 credits for attending the orientation session (which included the first survey and skills test), .5 credits each for 
completing the skills tests at time points 2 and 3 , and 1 credit each for completing the online surveys at time points 2 and 3 . Kinesiology students were made aware of the studies both by an in-class presentation conducted by the principal investigator, as well as by their instructors placing a link to information regarding the study information on the respective classes' blackboard website. The instructors for these courses compensated their students for completion of study participation through extra credit points in their respective classes. Each student who completed all phases of the study was given an increase of one letter grade on a final test or project in their respective class. Partial credit was also given to these participants for completing limited portions of the study by giving them a reduced number of extra credit points based on the length of participation.

These participants were, and will continue to be, protected through several avenues. Sources of identifiable data including informed consent forms and electronic spreadsheets were kept in a locked cabinet, behind a locked door, or protected with encryption and password. Only those individuals approved by the IRB to assist in research efforts were given access to the locked files. Coercion of participants was avoided by allowing students to have a free choice of whether to participate in this study or not, giving information about the study up front, providing alternative options in lieu of study participation (to obtain course credit or extra credit), and allowing participants to withdraw at any time from any study that was begun in good faith. Because some degree of exercise was required in this study, all participants who took place in physical activity for the purposes of this study were monitored during activity sessions by one of the trained research staff to ensure safety.

\subsection{Design}

The randomized experimental design consisted of a 3 (Introductory Training Method: None, Exergame, Traditional) X 3 (Time: Assessment 1, Assessment 2, Assessment 3) mixed design, to assess systematic changes in the dependent variables of physical activity, racquetball skills tests scores, self-efficacy for racquetball, enjoyment, future intention for 
physical activity, and activity center use for both general measures of physical activity and for the respective sport specifically. The independent variable of introductory training method was operationally defined as introducing participants to the sport of racquetball with training during weeks $0-4$ through an exergame, through traditional training in a racquetball court, or by introducing them to the sport through traditional means, but only requiring them to meet at the facility to study for classes during weeks $0-4$ (control group). The exergame group served as the experimental group, while the other groups served as controls. Participants were scheduled for twice weekly, 30-minute training sessions which took place over an 8 week training period. Weeks $0-4$ consisted of the introductory training methods assigned to each of the three different groups, which were followed by weeks $5-8$ where all groups proceeded to play the sport of racquetball on racquetball courts. During each training session for the duration of the study, all participants were fitted with an accelerometer to measure levels of activity performed during their sessions. Given the novelty of this model, the design allowed the researchers to confirm an appropriate effect of the exergame condition against a control that initiates training with no supplementary training. The within subjects factor of time consisted of three assessments, the first taken during orientation, the second taken during the transition phase between weeks 4 and 5, and the third taken upon conclusion of the required 8 week participation period.

\section{$\underline{2.3 \text { Procedures }}$}

The current study was conducted over two consecutive long semesters and the variable of semester was examined in the analyses to ensure that there were no seasonal differences in the physical activity outcomes. However, individual students only participated in the study over one long semester. Participants were recruited from the Psychology Human Subjects Participant Pool and from three professor-approved Kinesiology classes. Eligibility criteria for participants in the current study required them to be enrolled in the Psychology participant pool or in one of the three approved Kinesiology classes on campus, be at least 18 years of age 
(may have signed parental consent if 17 years old, not be a regular exerciser, be able to read, write and speak the English language, have little or no experience with racquetball, and have met minimum health requirements to perform physical activity. Exclusion criteria were not having been enrolled in the Psychology participant pool or one of the three approved Kinesiology classes on campus, under the age of 18 without parental consent, being a regular exerciser, not able to read, write and speak the English language, having significant experience with racquetball, and not meeting minimum health requirements to perform physical activity. All participants who wished to enroll in the study were first required to meet minimum criteria set in place by a prescreen questionnaire. This questionnaire was administered on the Psychology Participant Pool SONA system for the Psychology participants, and was administered through a link to a Google docs version of the questionnaire that was placed on the class-specific Blackboard page for the Kinesiology classes.

Pre-screening questions were used to ensure a sample that were not regular exercisers, had little knowledge or experience of racquetball, and met minimum health guidelines for engaging in physical activity (particularly screening for risk of cardiac event). These health requirements were mostly related to risk of cardiac event and inquired about things such as angina, shortness of breath at rest, fainting, blood pressure, past exercise, physician recommendations, smoking status, and diabetes. For the purposes of this study, sedentary was defined as those individuals that did not meet the minimum standard for exercise as detailed by the American College of Sports Medicine (Haskell et al., 2007). This minimum standard was defined as at least 20 minutes of vigorous physical activity for at least 3 days per week, or at least 30 minutes of moderately intense physical activity for at least 5 days per week. Participants who met the eligibility requirements were contacted within the Participant Pool system (Psychology) or through a participant-provided email address (Kinesiology) and invited to participate in the current project. Upon agreeing to participate, participants were scheduled for an orientation session convenient to their schedules. Participants then attended an 
orientation which reviewed the study, required the signing of a hard copy of the informed consent, fielded questions, provided the first assessment ( $\mathrm{T} 1$ ), and provided an introduction to the sport of racquetball. This orientation took place in a computer lab at the on-campus fitness center, consisted of no more than 8 individuals, and lasted approximately two hours. Participants were given 60 minutes to take the first assessment. Directly following completion of the first assessment, basic introduction of the sport of racquetball was provided in a traditional manner for all groups. This introduction included a training video recorded by professional racquetball coaches, diagrams of a racquetball court, a question and answer session, and a demonstration on an actual racquetball court. The orientation sessions were held in a meeting room in the on-campus activity center to allow participants to discover its location prior to the experimental sessions. After completion of the racquetball demonstration, participants were then required to take two racquetball skills tests (short-wall and long-wall volley tests). Completing these skills tests concluded orientation.

Following orientation, randomization of the participants into one of the three training groups was conducted. This procedure consisted of two layers of randomization, as the sample comprised primarily of females. Therefore, one layer randomized males equally into each group whereas the second randomized females into the groups, which ensured an equal representation of both genders in each group. Randomization was conducted using a random number table. The day of the week, starting with Monday as day 1 , determined the page of the book of random number tables to select. Then, a column and row was selected by the researcher by randomly dropping a pencil onto the page without looking. The following numbers were then read from left to right, where any value of 1,2 , or 3 would determine the placement of a participant as taken from a randomly sorted list of participant ID numbers. This procedure continued until all groups were filled equally and was conducted in the same fashion for both semesters in which the study took place. 
Participants were then contacted within two to five days of finishing orientation to schedule small groups of no more than six participants for the exergame and traditional training groups. Because six participants were used for each group, the use of three racquetball courts was necessary, as the participants were usually playing one-on-one against one another. During weeks $0-4$ of the experimental phase of the study, all groups were required to come to the campus activity center for 30-minute, twice weekly sessions. During each of these sessions, all participants were fitted with an accelerometer to measure the levels of activity they performed during their training sessions. These devices were pre-set to record measurements only during the participant's scheduled time, were about the size of a small pager or cell phone, and were worn just left or right of center on the waistband of the pants.

Training session content varied depending on study group assignment and week of the training. During Weeks 0-4, participants in the control group were instructed to only study or use the facility's computers during training sessions. However, those in the exergame group were instructed to play a racquetball exergame for a minimum of 20 minutes. Participants in the traditional training group were instructed to play supervised, singles games of racquetball, for at least 20 minutes during the twice weekly sessions. Unless hindered by scheduling or availability, those in the exergame and traditional training groups switched singles partners every session during Weeks 0 - 4 to promote novelty and adaptation, as well as control for skill discrepancies between players. Likewise, this practice continued for all groups during Weeks 5 $-8$.

Following the first four weeks of involvement, participants transitioned into the final four weeks of the study, which was initiated by completing the second assessment (T2). One of the measurements for the second assessment was the second racquetball skills test, which was administered just before the first weekly session during Week 5 . This skills test took approximately 15 minutes. The survey portion of the second assessment was administered online, took approximately 45- 60 minutes to complete, and was open for completion during a 
72 hour window. During Weeks $5-8$, the exergame and control groups were transitioned into actually playing racquetball in a similar manner as the traditional training group. The traditional training group simply continued their previous activities. Directly following the final weekly session during week 8, participants completed the last racquetball skills test, which also took approximately 15 minutes. After completion of all 8 weeks, the online final assessment was made available to the participants (T3). This survey was also made available online during a 72 hour window, and took approximately 45-60 minutes to complete. Reminders were sent to participants throughout the study with timely emails and phone calls regarding meetings and questionnaire obligations. Upon completion of the study, an optional, double-elimination racquetball tournament was held for those individuals in the study wishing to participate and took place on a single, scheduled date at the end of each semester.

\section{$\underline{2.4 \text { Measurements }}$}

Participants completed a battery of questionnaires before starting the eight weeks, between weeks 4 and 5, and immediately after the end. The initial assessment measured demographic and background data, general health, distance from the facility, social desirability, previous experience with video games (including this specific game), previous experience with sports (including racquetball), enjoyment, and previous participation in physical activity. All three assessments measured future intention to perform physical activity, self-efficacy to regulate exercise, self-efficacy for racquetball, stage of change, physical activity, and racquetball skills test performance. Activity counts from the accelerometers were collected during each of the 16 training sessions. Activity center use (card swipe data) was collected separately from the three assessments with the assistance of IRB-approved individuals from the fitness center staff. Assessment of participants' feedback ratings was also collected at the final time point. This final assessment took place after the participants' final session and in which they were given a 72 hour window to complete it online. 


\subsubsection{Demographics and Health}

The demographic survey asked participants for information about their age, gender, ethnicity/race, marital status, student status, income level, and employment status. Participants responded to the demographic questions by selecting categories or filling in open-ended spaces. General health items such as history of disease or injury, sickness frequencies, family medical history, history of surgery, and smoking and drinking behavior, were answered by allowing participants to choose categorical answers pertaining to the questions at hand. Participants' distance from the on-campus activity center was also calculated through Google Maps by using their address to find the approximate miles the participant lives away from the on-campus activity center.

\subsubsection{Previous Activity Experience}

The scale assessing previous video game participation began by asking the participant to rate their experience with video games in general through a Likert scale of 1 to 10 , where 1 is no experience, 5 is a moderate amount, and 10 is a large amount of experience. This question was then followed by several more specific questions that asked for experience ratings with sedentary video games, exergames, and the specific exergame used for this study. Previous engagement in sports, including racquetball, was reported by allowing participants to rate their experience with certain sports through a Likert scale of 1 to 10 , where 1 is no experience, 5 is a moderate amount, and 10 is a large amount of experience. This was then supplemented by items measuring fitness center use (including on-campus, and off-campus fitness centers). In these items, participants were able to select whether or not (yes/no) they used a fitness center and frequency of their use (Dougall, et al., 2011). Physical activity was measured using the Godin Leisure-Time Physical Activity Questionnaire (Cronbach's alpha = .76; Godin \& Shepard, 1997). This scale allowed participants to rate how often they engaged in leisure time physical activities by answering open-ended questions concerning how many days per week they engaged in vigorous, moderate, or light physical activity. Participants also reported how often 
they engaged in vigorous activities in a usual week by selecting a categorical option $(0=$ never/rarely, $1=$ sometimes, $2=$ often). Previous experience with physical activity and racquetball was taken at Assessment 1 to establish a baseline and to confirm prescreen criteria were met. The sport of racquetball was chosen specifically because it is a novel sport, for which the chances of participants having prior knowledge of the activity were minimal.

\subsubsection{Racquetball Skills Testing}

Racquetball skills improvement was measured by comparing performance scores on the short-wall volley test and the long-wall volley test across time points (Safrit, 1990). These tests were selected for their simplicity, validity, reliability (short-wall: college women: $R_{x x}=.86$, college men: $R_{x x}=.76$; long-wall: college women: $R_{x x}=.82$, college men: $R_{x x}=.85$ ), and use of an array of racquetball skills. These tests required the player to volley the racquetball off of the front wall and return it as many times as possible inside a 30-second window from both a short distance and a long distance. Scores for these tests consisted of the total number of legal returns inside of the 30 -second windows. Values for this measurement were calculated as the sum of the scores from both tests.

\subsubsection{Activity Center Use}

In addition to survey measures, use of the campus activity center was collected every time each participant entered through the facility's turnstiles by using their student I.D. card or computer keypad pass code. All entries related to the study, as well as participant-reported entries not for exercise, were estimated and subtracted from these measures. With the permission of the participants, card swipes for the entire duration of the study were collected at the end of each semester's study period. Activity center staff, who were part of the protocol, aided researchers in retrieving these data from the computer system at the activity center itself. Once these data were retrieved, they were compiled into a database and then further compiled into a single time point variable that accounted for the total number of times each participant entered the activity center during their respective semester of participation. This value was then 
adjusted using the participant's estimate of the percentage of time they entered the activity center specifically to exercise. This final value was then an estimate of the frequency of use of the activity center for exercise purposes to be compared between training groups in the analyses.

\subsubsection{Self-efficacy for Racquetball}

Self-efficacy for racquetball specifically was measured by modifying the Racquetball Skills Test Battery (Lam \& Zhang, 2002) while using the procedures followed by the Selfefficacy Scale for Quad Rugby Skills (SEQRS) (Adnan, McKenzie, \& Miyahara, 2001) as a guide. The current study created a measure of sport-specific self-efficacy by taking the individual skills needed to complete a skills test battery designed for the sport of racquetball. Players were introduced to the skills test battery and informed about the cognitive and physical aspects it required them to perform. Before actually performing the skills tests, the players completed the Self-efficacy for Racquetball questionnaire. Although the players needed only to think they would have to perform the skills tests, this method has been shown to effectively measure the players belief in their ability (self-efficacy) to complete the individual skills involved in the skills test battery. Following the design of the SEQRS, the Self-efficacy for Racquetball scale was created using the individual skills in the Racquetball Skills Test Battery. This skills test battery is particularly salient as it was designed specifically for young, adult beginners and has been proven both valid and reliable ( $G$ coefficient $=.61-.84)$ (Lam \& Zhang, 2002). Participants rated how confident they were in performing the individual skills (e.g., forehand power drive, ceiling shot) used in racquetball on a Likert scale of 1 to 5 , where 1 is not at all confident and 5 is extremely confident.

\subsubsection{Enjoyment of Racquetball}

Sport enjoyment, specific to racquetball, was measured using the Sport Enjoyment subscale within the Sport Commitment Model (Cronbach's alpha= .95; Scanlan, Carpenter, Schmidt, Simons, \& Keeler, 1993). Past studies have successfully modified this scale to reflect 
a different specific sport, where enjoyment of the sport of tennis was found to be the best predictor of commitment to play (Casper \& Stellino, 2008). For this study, the specific sport in question was modified for racquetball. Items within this scale asked participants if they enjoyed, liked, had fun, and were happy playing the sport of racquetball. Scoring of the enjoyment scale involved taking an average of the four items of which the scale consists. This average was taken at all three time points.

\subsubsection{Future Intention for Physical Activity}

Future intention to perform physical activity was measured by having participants rate the likelihood in which they would engage in physical activities in the near future using a Likert scale of 1 to 10 ( $1=$ will not do at all, $5=$ possibly will do, 10=highly certain will do). This scale was modified for future intention from the Godin Leisure-Time Questionnaire (Godin \& Shepard, 1997).

\subsubsection{Self-efficacy for Exercise}

Self-efficacy to regulate exercise was measured with the Self-efficacy to Regulate Exercise and Sports questionnaire using a Likert scale which allowed participants to choose from 1 to $10(1=$ not at all likely, 10 = very likely $)$ how likely they were to overcome certain exercise barriers (Cronbach's alphas $=0.93-0.94$; Bandura, 2006).

\subsubsection{Stage of Change}

The measurement for Stage of Change (Prochaska, DiClemente, \& Norcross, 1992) used a scale that allowed for the determination of stage of change by having participants choose the categorical definitions which most closely matched their behaviors (Dougall, et al., 2011). The definitions stated "I have not considered doing this or do not wish to at this time" (pre-contemplation stage), "I have considered doing this but have not yet done so" (contemplation stage), "I am doing this and have considered doing this regularly but I am not currently doing this regularly" (preparation stage), "I have recently begun doing this regularly" 
(action stage), "and I am currently doing this regularly and plan to continue to do so" (maintenance stage).

\subsubsection{Racquetball Participation}

Frequencies of racquetball participation were reported by participants using an openended question. This question was a single item that asked participants how many games of racquetball they had played during the last 4 weeks, not including the games played for study participation.

\subsubsection{Activity Levels}

Past research has found that levels of activity during exergame use can be effectively measured using an accelerometer (Lanningham-Foster; et al., 2009). This instrument measures activity through changes in acceleration due to movement. The device for the current study was worn on the waistband and was about the size of a small pager. Due to its small size, light weight, and unobtrusive nature, accelerometers have been frequently used while measuring movement during physical activity, and can provide a feasible and accurate way to compare activity levels between groups during exergame use and on the racquetball court. Actitrainer accelerometers units were used for the current study. These devices measured small changes in movement in the form of milli-G's, where a $G$ is the force of gravity and a unit increase measures how much the force of gravity is multiplied due to changes in motion. Data collected from accelerometers consisted of activity based measures including steps taken and calories burned in addition to the three measures of activity based on accelerometer counts. These measures calculated G's in two directions (forward/backward and side to side) as well a total measure of activity that does not take into account direction of movement. Actitrainer activity measures were uploaded to a research computer via USB port after each day of training sessions was completed. The information was displayed in Microsoft excel format, where it documented the activity for every minute, of every training session attended, for each of the participants. Upon study completion, these individual excel files were compiled into an excel 
database, modified for export, and then exported to SPSS for statistical analysis. Within SPSS, a variable was created for each minute activity was scheduled to take place. This was repeated for all activity measures attained by the accelerometers. For the purposes of the analyses run for the current study, total activity as measured by accelerometer counts was averaged to obtain weekly values for each participant.

\subsubsection{Participant Feedback}

Lastly, a questionnaire asked participants to give feedback about the study and consisted of categorical and open-ended questions that had participants respond to how they would have improved the study and any comments they wished to report. 


\section{CHAPTER 3}

\section{RESULTS}

\section{$\underline{3.1 \text { Recruitment }}$}

While condition-based training was carried out successively, attrition was seen between all three time points (see Figure 3.1). A total of 103 participants were recruited and subsequently completed T1. Of this total, 9 participants were lost to attrition before T2, with a single case completing only the questionnaire portion of the second assessment before dropping out. All other participants completed all time points with the exception of a single participant missing the skills test at T2. Thus, 93 participants were considered completers, with a total of 9 lost to attrition, and 1 participant missing a single skills test. Therefore, overall attrition in this sample was minimal (9\%) and far below levels reported with other exercise interventions among college students (18 - 34\%; Linke, 2011). 


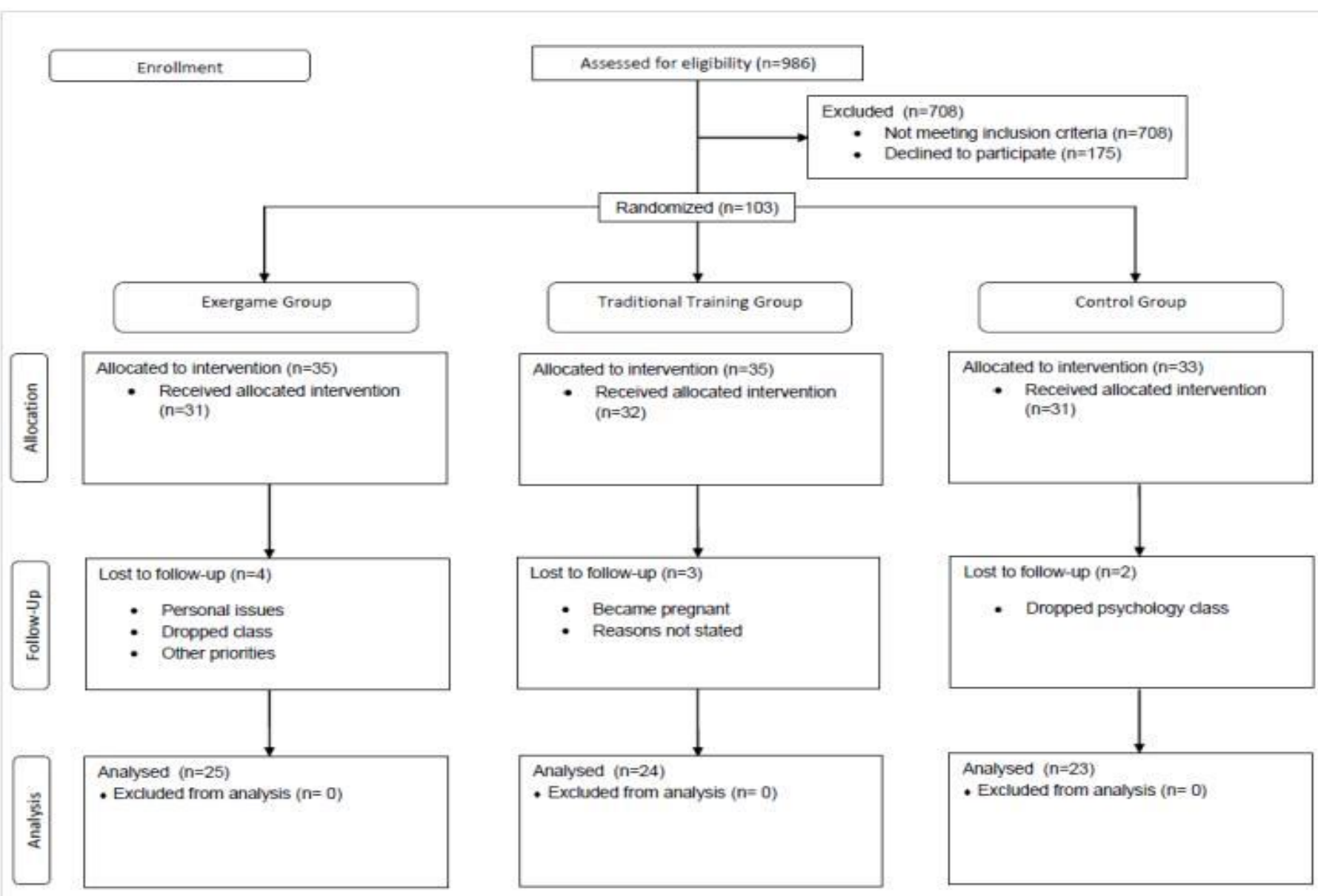

Figure 3.1 Participant Flow Chart 
Participants were recruited for the present study using the Psychology Department Participant Pool as well as three professor-approved classes within the Kinesiology department. All participants were recruited within the first seven weeks of each semester, for both fall of 2011 and spring of 2012 (August - October for fall and January - March for spring). Participants from the Psychology participant pool were all students taking courses within the Psychology Department which required research study participation for course completion, or for extra credit points within the class. Participants recruited from Kinesiology were all students taking at least one of three courses approved for recruitment by the instructor of record, as well as by the University's IRB. These students were allowed to participate in a number of different local research studies in exchange for extra credit points based on the difficulty of the study and the participant's length of involvement. Between training groups, differences of baseline physical activity levels and income were found, but were all very minor in nature. As the sample was primarily female, randomization techniques were used ensure that each group held a balanced number of males and females. Thus, this was carried forward for each time point (see Tables 3.1 and 3.2). 
Table 3.1 Demographic Characteristics by Training Group.

\begin{tabular}{|c|c|c|c|c|c|c|c|}
\hline \multirow[t]{2}{*}{$\begin{array}{l}\text { Demographic } \\
\text { Characteristic }\end{array}$} & \multicolumn{2}{|c|}{ Exergame } & \multicolumn{2}{|c|}{$\begin{array}{l}\text { Traditional } \\
\text { Training }\end{array}$} & \multicolumn{2}{|c|}{ Control } & \multirow[b]{3}{*}{$F(2,98)=.37, p=.691, \eta_{\mathrm{p}}^{2}=.01$} \\
\hline & $n$ & $M(S E)$ & $n$ & $M(S E)$ & $n$ & $M(S E)$ & \\
\hline Age & 35 & $\begin{array}{l}21.00 \\
(.80)\end{array}$ & 34 & $\begin{array}{l}21.35 \\
(.81)\end{array}$ & 32 & $21.98(.83)$ & \\
\hline \multirow[t]{2}{*}{ BMI } & 31 & $\begin{array}{l}25.50 \\
(.98)\end{array}$ & 34 & $\begin{array}{l}23.81 \\
(.94)\end{array}$ & 33 & $24.88(.95)$ & $F(2,98)=.80, p=.453, \mathrm{n}_{\mathrm{p}}^{2}=.02$ \\
\hline & $n$ & $\%$ & $n$ & $\%$ & $n$ & $\%$ & \\
\hline Gender & & & & & & & $\mathrm{X}^{2}(2, N=102)=.03, p=.986, w=.02$ \\
\hline Male & 11 & $31.4 \%$ & 11 & $32.4 \%$ & 11 & $33.3 \%$ & \\
\hline Female & 24 & $68.6 \%$ & 23 & $67.6 \%$ & 22 & $66.7 \%$ & \\
\hline Ethnicity & & & & & & & $X^{2}(2, N=96)=2.94, p=.230, w=.21$ \\
\hline $\begin{array}{l}\text { Not } \\
\text { Hispanic }\end{array}$ & 18 & $54.5 \%$ & 23 & $74.2 \%$ & 22 & $68.8 \%$ & \\
\hline Hispanic & 15 & $45.5 \%$ & 8 & $25.8 \%$ & 10 & $31.3 \%$ & \\
\hline Race & & & & & & & $X^{2}(8, N=100)=8.41, p=.395, w=.29$ \\
\hline Caucasian & 16 & $47.1 \%$ & 11 & $32.4 \%$ & 12 & $37.5 \%$ & \\
\hline Black & 5 & $14.7 \%$ & 10 & $29.4 \%$ & 9 & $28.1 \%$ & \\
\hline Asian & 5 & $14.7 \%$ & 7 & $20.6 \%$ & 4 & $12.5 \%$ & \\
\hline $\begin{array}{l}\text { Native } \\
\text { American/ } \\
\text { Alaskan }\end{array}$ & 0 & $0 \%$ & 0 & $0 \%$ & 2 & $6.3 \%$ & \\
\hline $\begin{array}{r}\text { Other } \\
\text { Employed }\end{array}$ & 8 & $23.5 \%$ & 6 & $17.6 \%$ & 5 & $15.6 \%$ & \\
\hline & & & & & & & $X^{2}(2, N=103)=3.32, p=.19, w=.21$ \\
\hline Yes & 16 & $45.7 \%$ & 22 & $62.9 \%$ & 14 & $42.4 \%$ & \\
\hline $\begin{array}{l}\text { No } \\
\text { Household }\end{array}$ & 19 & $54.3 \%$ & 13 & $37.1 \%$ & 19 & $57.6 \%$ & \\
\hline $\begin{array}{c}\text { Income } \\
\text { Under } \\
\$ 10,000 \\
\end{array}$ & 3 & $8.8 \%$ & 3 & $8.6 \%$ & 5 & $15.2 \%$ & $X^{2}(14, N=102)=25.02, p=.034, w=.79 \dagger$ \\
\hline
\end{tabular}


Table 3.1 Continued

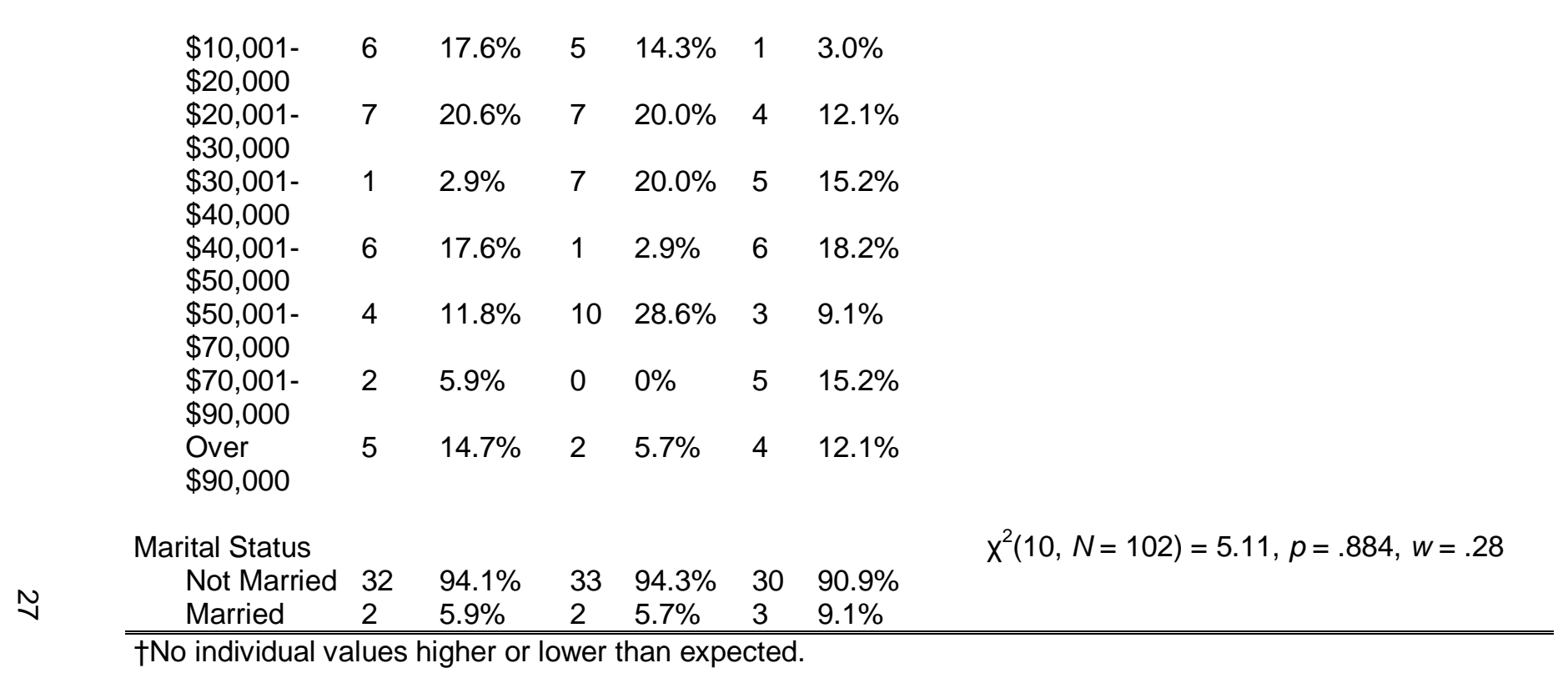


Table 3.2 Training Group Means and Standard Errors for Hypothesis 1 Outcomes at Week 0 - Baseline (T1), After Week 4 - Transition (T2), and After Week 8 - Completion of Training (T3).

\begin{tabular}{|c|c|c|c|c|c|c|c|c|c|c|c|c|}
\hline \multirow{2}{*}{ Variable } & \multicolumn{3}{|c|}{ Exergame } & \multicolumn{3}{|c|}{ Traditional } & \multicolumn{3}{|c|}{ Control } & \multicolumn{3}{|c|}{ Total Sample } \\
\hline & $n$ & $M$ & $(S E)$ & $n$ & $M$ & $(S E)$ & $n$ & $M$ & $(S E)$ & $n$ & $M$ & $(S E)$ \\
\hline Skills Test & 30 & & & 31 & & & 30 & & & 91 & & \\
\hline $\begin{array}{l}\text { T1 } \\
\text { T2 } \\
\text { T3 }\end{array}$ & & $\begin{array}{r}29.96^{1} \\
32.67^{2} \\
3827^{a ; 1,2}\end{array}$ & $\begin{array}{l}1.48 \\
1.56 \\
172\end{array}$ & & $\begin{array}{r}31.29^{1} \\
35.83^{\mathrm{b} ; 2,1} \\
38.99^{\mathrm{b} ; 1,2}\end{array}$ & $\begin{array}{l}1.45 \\
1.54 \\
1.69\end{array}$ & & $\begin{array}{r}29.37^{1} \\
29.86^{a, b} \\
33.63^{a, b ; 1}\end{array}$ & $\begin{array}{l}1.47 \\
1.56 \\
171\end{array}$ & & $\begin{array}{r}30.21^{1} \\
32.79^{2} \\
36.96^{1,2}\end{array}$ & $\begin{array}{r}.88 \\
.93 \\
1.03\end{array}$ \\
\hline Total & & 33.63 & 1.44 & & $35.37^{\mathrm{b}}$ & 1.42 & & $30.96^{\mathrm{b}}$ & 1.44 & & & \\
\hline
\end{tabular}

\section{Racquetball} self-efficacy

T1
T2
T3
Total

T2 Total

General self-efficacy

T1
T2
T3
Total

Enjoyment†

T1

T2

T3
31

$$
\begin{array}{rr}
2.57^{1} & 0.13 \\
3.01^{\mathrm{a}, 1,2} & 0.14 \\
3.64^{1,2} & 0.12 \\
3.07 & 0.11
\end{array}
$$

31

$$
\begin{array}{rr}
2.87^{1} & 0.13 \\
3.72^{\mathrm{a}, \mathrm{b} ; 1,2} & 0.14 \\
4.03^{1,2} & 0.12 \\
3.54^{\mathrm{b}} & 0.11
\end{array}
$$

\section{0}

$$
\begin{array}{rr}
2.73^{1} & 0.13 \\
2.84^{\mathrm{b} ; 2} & 0.14 \\
3.73^{1,2} & 0.12 \\
3.10^{\mathrm{b}} & 0.11
\end{array}
$$

92

$$
\begin{array}{rr}
2.72^{1} & 0.08 \\
3.19^{2} & 0.08 \\
3.80^{1,2} & 0.07
\end{array}
$$

31

$\begin{array}{ll}5.24 & 0.34 \\ 5.31 & 0.35 \\ 6.01 & 0.37 \\ 5.52 & 0.31\end{array}$

$\begin{array}{ll}5.52 & 0.31\end{array}$

30

$$
\begin{array}{rr}
19.64^{1} & 1.10 \\
19.09^{2} & 1.13 \\
22.50^{1,2} & 0.85 \\
20.41 & 0.88
\end{array}
$$

$\begin{array}{ll}5.65 & .34 \\ 5.96 & .35 \\ 6.46 & .37 \\ 6.02 & .31\end{array}$

31

$\begin{array}{ll}19.44^{1} & 1.07 \\ 22.21^{\mathrm{b}} & 1.11 \\ 23.17^{1} & 0.84\end{array}$

31

$\begin{array}{ll}5.67 & .33 \\ 5.85 & .34 \\ 5.90 & .37 \\ 5.81 & .31\end{array}$

30

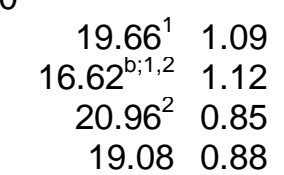

93

$$
\begin{array}{ll}
5.52 & 0.20
\end{array}
$$$$
\begin{array}{lll}
5.71 & 0.21
\end{array}
$$$$
\begin{array}{lll}
6.12 & 0.22
\end{array}
$$

91

$$
\begin{array}{rr}
19.58^{1} & 0.65 \\
19.31^{2} & 0.67 \\
22.21^{1,2} & 0.51
\end{array}
$$

†Squared transformation applied to normalize distribution. Note: Means in the same row with the same superscript letter are significantly different. Means in the same column within the same group that have the same superscript number are significantly different. 
Baseline differences in sociodemographic characteristics were examined between genders and the department from which a participant was recruited (Psychology/Kinesiology). Males had higher baseline levels of self-efficacy for racquetball (Males: $M=2.90, S E=.13$; Females: $M=2.54, S E=.09$ ) and had more sports experience (Males: $M=3.59, S E=.21$; Females: $M=3.09, S E=.14)$ than did females, $F(1,102)=5.60, p=.020$, partial $\eta^{2}=.05$ and $F(1,102)=3.82, p=.053$, partial $\eta^{2}=.04$, respectively. Additionally, males were also found to have higher levels of previous experience with video games (Males: $M=4.71, S E=.28$; Females: $M=3.72, S E=.19)$ than did females, $F(1,102)=8.34, p=.005$, partial $\eta^{2}=.08$. These gender differences were consistent with those expected from the review of past literature (De Moor, et al., 2007, Edwards, Bocarro, Kanters, \& Casper, 2011, \& Lam, Sit, \& McManus, 2011). Participants recruited from the Kinesiology Department showed slightly higher levels of baseline physical activity (Kinesiology: $M=43.61, S E=4.65$; Psychology: $M=32.75, S E=$ 3.84), self-efficacy for general physical activity (Kinesiology: $M=6.01, S E=.30$; Psychology: $M$ $=5.09, S E=.25)$, and were slightly older (Kinesiology: $M=22.89, S E=.72 ;$ Psychology: $M=$

$20.48, S E=.58)$ than those from Psychology, $F(1,101)=3.24, p=.075$, partial $\eta^{2}=.03, F(1$, $101)=5.59, p=.020$, partial $\eta^{2}=.05$ and $F(1,101)=6.77, p=.011$, partial $\eta^{2}=.06$, respectively. Because Kinesiology often studies the science of movement and exercise, it was also logical to see several of these differences. However, all participants, regardless of department, were not regular exercisers at the time of enrollment.

\subsection{Data Screening}

The descriptive statistics of the variables involved in testing the hypotheses can be found in Table 3.1. Frequency, descriptive, and distribution data were inspected for all outcome variables to insure plausible means and standard deviations, proper filling of cells for testing, and normal distributions. Skewness statistics and histograms were examined to consider variable distribution. These tests found a negatively skewed distribution for enjoyment and positively skewed distributions for general physical activity (self-report, Godin) and fitness 
center use. Transformations for these variables were necessary, resulting in a squared transformation being applied to enjoyment, a square root transformation being applied to general physical activity and a log transformation being applied to fitness center use. These data were also checked for the assumptions required by their respective statistical models.

\section{$\underline{3.3 \text { Statistical Results }}$}

Analyses testing Hypotheses 1 and 2 were conducted using analysis of covariance (ANCOVA) models. Time was used as a predictor variable, as the majority of physical and cognitive physical activity outcomes were measured at multiple time points, where fitness center use was the only outcome measured at only one time point. Skills test performance, enjoyment, exercise self-exercise, racquetball self-efficacy, general physical activity (self-report, Godin) and future intention were all measured at T1, T2 and T3, whereas activity (as measured by accelerometer counts) was measured at every training session and then averaged for eight, weekly measurements for the purposes of analysis. Covariate and predictor variables for these models were selected by examining relationships among the data for the current study and by reviewing previous relevant literature. Using these criteria, the participant pool (Psychology/Kinesiology) from which a participant was recruited was included as a covariate in all ANCOVA models, as group differences were discovered. The variable of gender was included as a predictor in each of these models as it was expected to have an influence on the outcome variables. Furthermore, previous research has demonstrated the propensity of males to have a greater organic ability for physical activity than females (De Moor et al., 2007). Thus, including gender as a predictor variable improved the predictive ability of the models and allowed for the examination of possible moderation relationships. These models were subsequently probed using post hoc tests with Bonferroni corrections and all alpha criteria cutoffs were set at $\alpha=.05$. All analyses for the current study were conducted as intent-to-treat as is standard practice for analyzing data from randomized controlled trials. Therefore, all participants were included in the analyses regardless of whether or not they attended all of the 
study intervention sessions. This allows for a more conservative test of the models and better reflects the impact of the intervention in a non-research setting.

\subsubsection{Hypothesis 1}

The first prediction, stating that participants initially introduced to an exergame would report greater or equal performance on skills tests, activity (as measured by an accelerometer) and self-efficacy for the specific sport (racquetball) than those in the traditional training group, greater performance altogether than those in the control group, and greater enjoyment than all other groups across all time points, was analyzed using a repeated measures ANCOVA for each of the variables. Means, standard errors, and sample sizes for the outcome variables used in Hypothesis 1 can be found in Table 3.2.

For the variable of sports-specific skills tests, significant main effects of time, training group and gender were seen, $F(2,168)=19.22, p<.001$, partial $\eta^{2}=.19, F(2,84)=3.20, p=$ .046 , partial $\eta^{2}=.07$ and $F(1,84)=25.65, p<.001$, partial $\eta^{2}=.23$, respectively. Subsequent inspection of pairwise comparisons found that skills test scores increased at every time point for each of the training groups, that males $(M=37.76, S E=1.43)$ had higher scores than did females $(M=28.91, S E=1.00)$ overall, and that the traditional training group exhibited higher scores than the control group. Interactions within the model found significance with time by group and time by group by gender, $F(4,168)=4.36, p=.002$, partial $\eta^{2}=.09$ and $F(4,168)=$ 4.27, $p=.003$, partial $\eta^{2}=.09$, respectively. However, the interactions of group by gender and time by gender failed to reach significance, $F(2,84)=.77, p=.469$, partial $\eta^{2}=.02$ and $F(2$, $168)=.47, p=.624$, partial $\eta^{2}=.01$, respectively. Post hoc analysis of significant interactions showed that all groups performed better at T3 than T1, and that the exergame group showed higher scores at T3 than T1, whereas the traditional training performed better with each successive time point (see Figure 3.2). Moreover, time by group post hocs also showed that the traditional training group performed better than the control group at T2 and both this group and the exergame group (marginal, $p=.056$ ) performed better than the control group at T3.Pairwise 
comparisons for time by group by gender (see Figure 3.3) found that females in all groups performed better at T3 than all other time points, that females in the exergame and traditional training groups also performed better at T2 than at T1, and that males followed a similar pattern, but only for the exergame and traditional training groups. Gender group differences in these post hocs revealed that males exhibited higher skills test scores at all times points with the exception of the control group at T3. Additionally, findings that all other groups performed better than the control group at T3 were found to be a result of the males performance for both the exergame (marginal, $\mathrm{p}=.057$ ) and traditional training groups.

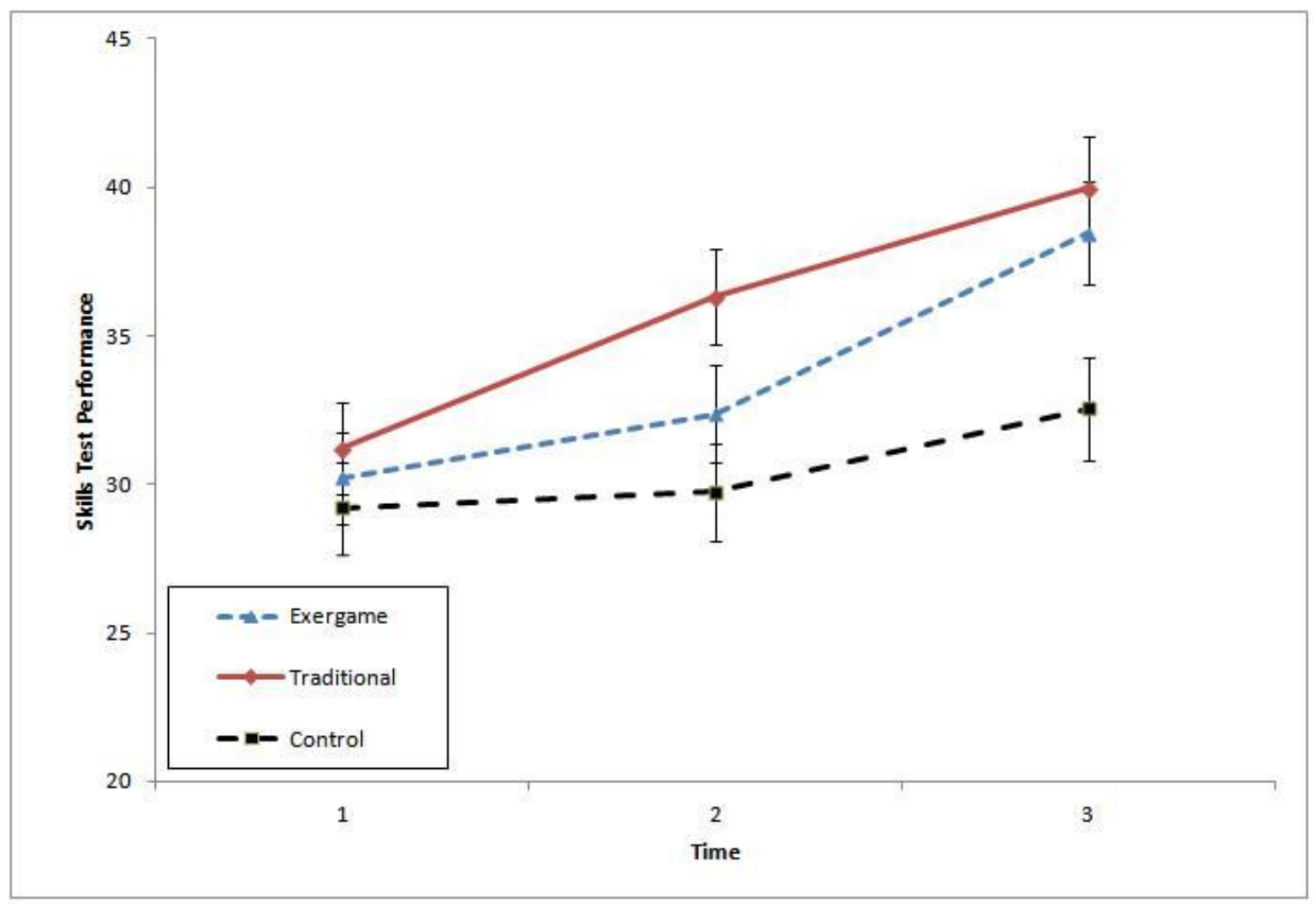

Figure 3.2 Skills Test Performance for the Significant Interaction of Time by Training Group. 


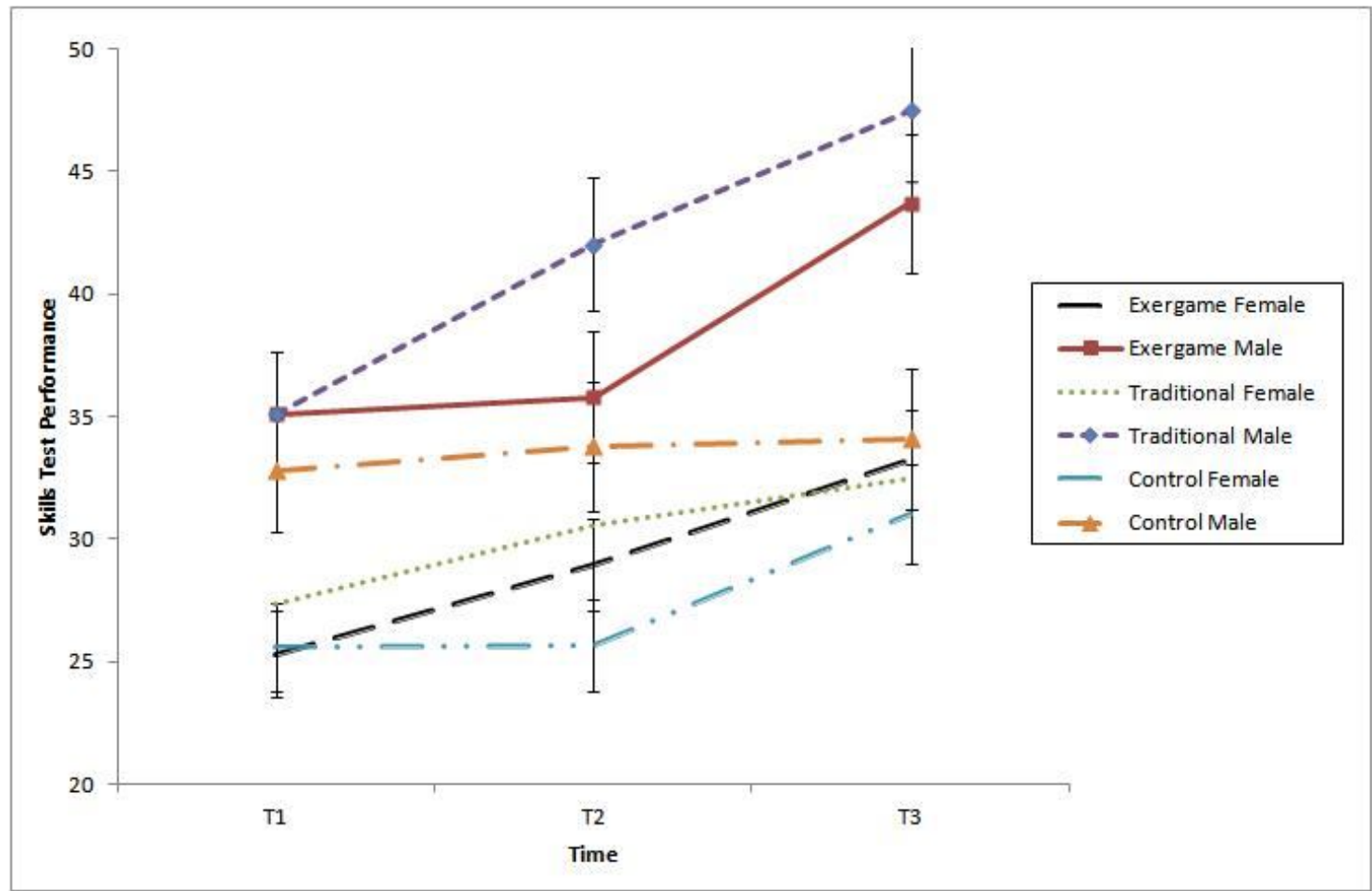

Figure 3.3 Skills Test Performance for the Significant Interaction of Time by Training Group by Gender.

The ANCOVA model for the variable of activity (as measured by accelerometer counts) was found to violate Mauchly's test of sphericity, thus resulting in the use of the GreenhouseGeisser test for correction. The subsequent results found main effects of time, training group and gender, $\mathrm{F}(4.67,275.45)=91.17, p<.001$, partial $\eta^{2}=.61, \mathrm{~F}(2,59)=61.87, p<.001$, partial $\eta^{2}=.68$ and $F(1,59)=9.53, p=.003$, partial $\eta^{2}=.14$, respectively. Subsequent pairwise comparisons for the significant main effect of time showed higher accelerometer counts for the last four weeks (see Table 3.3) for activity means and standard errors) than for the first four weeks as well Week 6 displaying higher activity than Week 5. Group differences were also found in that the traditional training group displayed higher activity counts than both the exergame and control groups, whereas gender differences showed that males $(M=2345.73$, $S E=90.89)$ recorded more activity than females $(M=2007.09, S E=60.43)$, overall. 
Additionally, significant interactions of time by group and time by group by gender were also discovered, $\mathrm{F}(9.34,275.45)=56.29, p<.001$, partial $\eta^{2}=.66$ and $F(9.34,275.45)=2.27, p=$ .017 , partial $\eta^{2}=.07$, respectively. Significant effects were not found for group by gender or time by gender interactions, $F(2,59)=.23, p=.799$, partial $\eta^{2}=.01$ and $F(4.67,275.45)=1.86, p=$ .107 , partial $\eta^{2}=.03$, respectively. Pairwise comparisons for the time by group interaction (see Figure 3.4) revealed that during Weeks 1-4 the traditional training group was more active than both the exergame and control groups. Additionally, the exergame and control groups had been significantly more active during Weeks 5-8 than during Weeks 1-4. Pairwise comparisons for the three-way interaction (see Figure 3.5) showed marked increases in activity levels during Weeks $5-8$ from Weeks $1-4$ for both genders of the exergame and control groups, with an additional result showing Week 6 activity above Week 5 activity specifically in control group females. While the traditional training group did not exhibit this same pattern, they did show increased activity levels during Weeks 3, 6 and 7 when compared to Week 5. Moreover, the traditional training group recorded greater activity levels than all other groups during Weeks 1 4 , but this finding was for females only. Males, however, were seen to perform more activity than females during Weeks 1,2 , and 4 in the traditional training group, whereas a similar finding was discovered during Weeks 6, 7 and 8 for the exergame group. 
Table 3.3 Training Group Means and Standard Errors for Activity for Hypothesis 1 at Weeks $1-8$.

\begin{tabular}{|c|c|c|c|c|c|c|c|c|c|}
\hline \multirow[t]{2}{*}{ Variable } & \multicolumn{2}{|l|}{ Exergame } & \multicolumn{2}{|c|}{ Traditional } & \multicolumn{2}{|l|}{ Control } & \multicolumn{3}{|c|}{ Total Sample } \\
\hline & $M$ & $(S E)$ & $M$ & $(S E)$ & $M$ & $(S E)$ & $n$ & $M$ & $(S E)$ \\
\hline Activity & 24 & & 22 & & 20 & & 66 & & \\
\hline Wk1 & $581.37^{\mathrm{a}, 1}$ & 87.47 & $3042.57^{\mathrm{a}, \mathrm{b}}$ & 90.49 & $642.06^{\mathrm{b} ; 1}$ & 97.54 & & $1422.00^{1}$ & 55.52 \\
\hline Wk2 & $515.88^{\mathrm{a} ; 2}$ & 102.20 & $3012.55^{\mathrm{a}, \mathrm{b}}$ & 105.77 & $474.31^{\mathrm{b} ; 2}$ & 113.97 & & $1334.25^{2}$ & 64.88 \\
\hline Wk3 & $499.25^{\mathrm{a} ; 3}$ & 104.64 & $3050.65^{\mathrm{a}, \mathrm{b}}$ & 108.26 & $568.58^{\mathrm{b} ; 3}$ & 116.69 & & $1372.83^{3}$ & 66.42 \\
\hline Wk4 & $551.16^{\mathrm{a}, 4}$ & 84.02 & $2928.22^{\mathrm{a}, \mathrm{b}}$ & 86.92 & $524.42^{\mathrm{b} ; 4}$ & 93.69 & & $1334.60^{4}$ & 53.33 \\
\hline Wk5 & $3107.58^{1,2,3,4}$ & 145.65 & $2631.92^{1}$ & 150.69 & $2690.10^{1,2,3,4}$ & 162.43 & & $2809.86^{1,2,3,4}$ & 92.46 \\
\hline Wk6 & $3207.17^{1,2,3,4}$ & 144.23 & $3199.50^{1}$ & 149.22 & $3042.81^{1,2,3,4}$ & 160.84 & & $3149.82^{1,2,3,4}$ & 91.56 \\
\hline Wk7 & $3069.62^{1,2,3,4}$ & 154.56 & 3142.26 & 159.90 & $2800.44^{1,2,3,4}$ & 172.36 & & $3004.11^{1,2,3,4}$ & 98.11 \\
\hline Wk8 & $3028.76^{1,2,3,4}$ & 156.91 & 3035.08 & 162.34 & $3026.74^{1,2,3,4}$ & 174.98 & & $3030.19^{1,2,3,4}$ & 99.61 \\
\hline Total & $1820.10^{\mathrm{a}}$ & 83.36 & $3005.34^{\mathrm{a}, \mathrm{b}}$ & 86.24 & $1721.18^{\mathrm{b}}$ & 92.96 & & & \\
\hline
\end{tabular}

Note: All activity scores measured by accelerometer counts. Means in the same row with the same superscript letter are significantly different. Means in the same column within the same group that have the same superscript number are significantly different. 


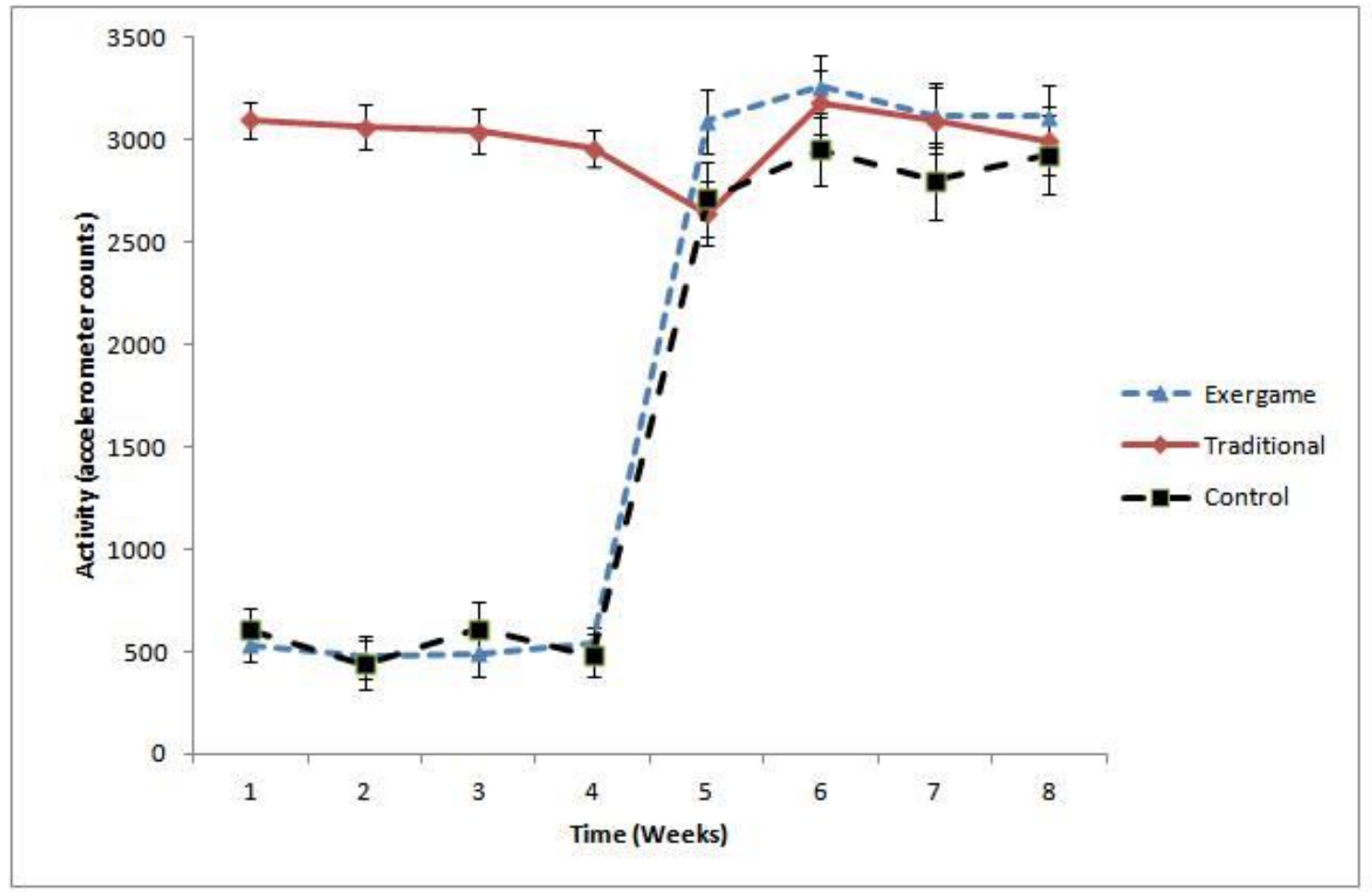

Figure 3.4 Activity (accelerometer counts) for the Significant Interaction of Time by Training Group. 


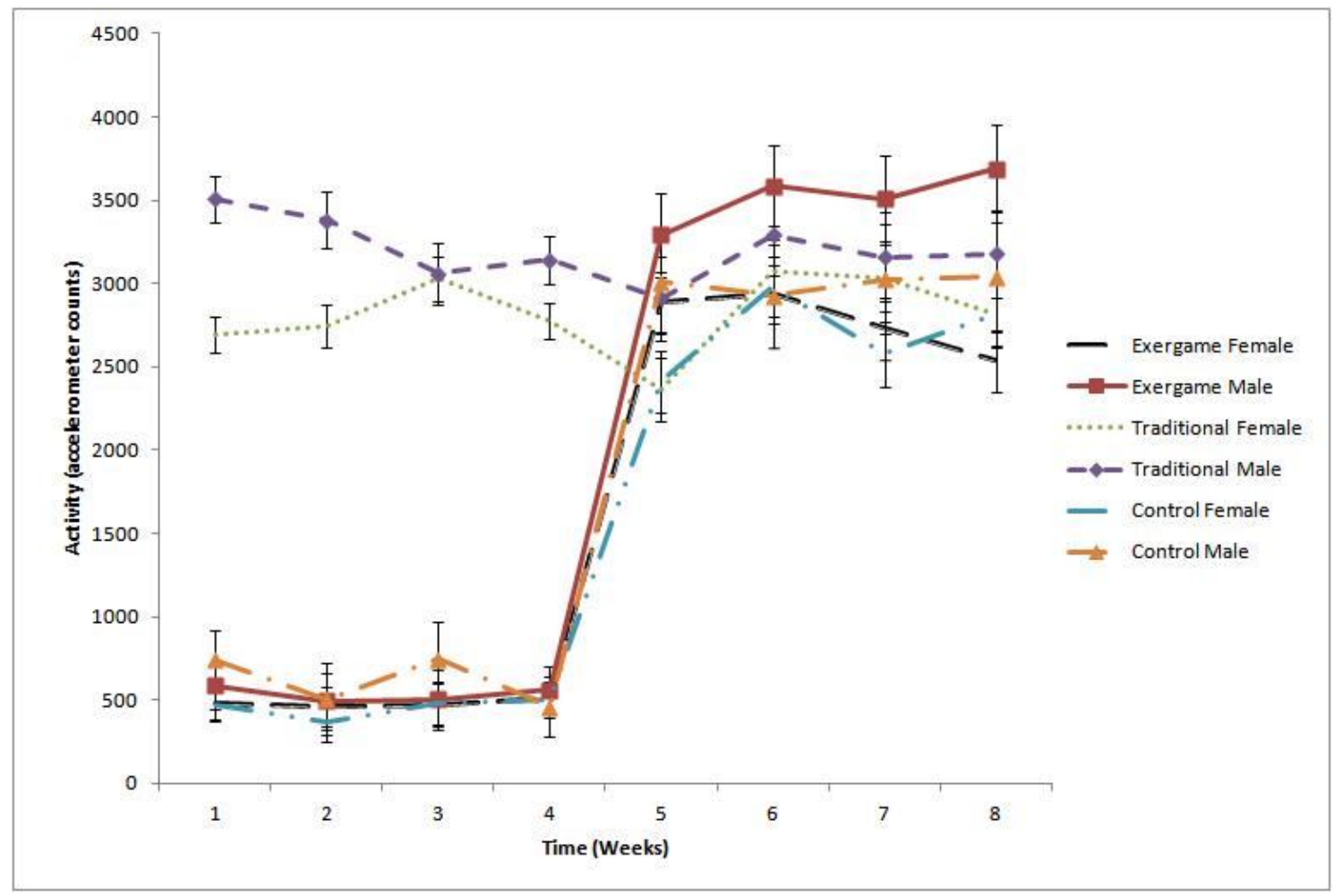

Figure 3.5 Activity (accelerometer counts) for the Significant Interaction of Time by Training Group by Gender.

Analysis of the mean differences in the racquetball self-efficacy scale was conducted using the Greenhouse-Geisser test correction, as the assumption of sphericity had been violated. The results for these tests produced main effects of time, group and gender, $F(1.76$, $149.95)=74.36, p<.001$, partial $\eta^{2}=.47, F(2,85)=4.03, p=.021$, partial $\eta^{2}=.09$, and $F(1$, $85)=7.19, p=.009$, partial $\eta^{2}=.08$, respectively. Post hoc testing showed that scores increased across time, the traditional training group displayed higher scores than all other groups, and that males $(M=3.42, S E=.11)$ had higher overall scores than did females $(M=3.06, S E=.07)$. Interactions of time by group and time by gender (marginal) were also found, $F(3.53,149.95)=$ 4.36, $p=.004$, partial $\eta^{2}=.09$ and $F(1.76,149.95)=2.88, p=.066$, partial $\eta^{2}=.03$, respectively. Additionally, the interactions of group by gender and time by group by gender were not found to be significant, $F(2,85)=1.02, p=.367$, partial $\eta^{2}=.02$ and $F(3.53,149.95)=1.63, p=.177$, 
partial $\eta^{2}=.04$, respectively. Post hoc analyses for significant interactions revealed that racquetball self-efficacy increased within each group across all time points with the exception of the control group from T1 to T2. Post hocs also showed that the traditional training group exhibited higher scores than all other groups, but only at T2 (see Figure 3.6). Furthermore, it was also seen that males reported more racquetball self-efficacy than females at T1 and T2 (see Figure 3.7).

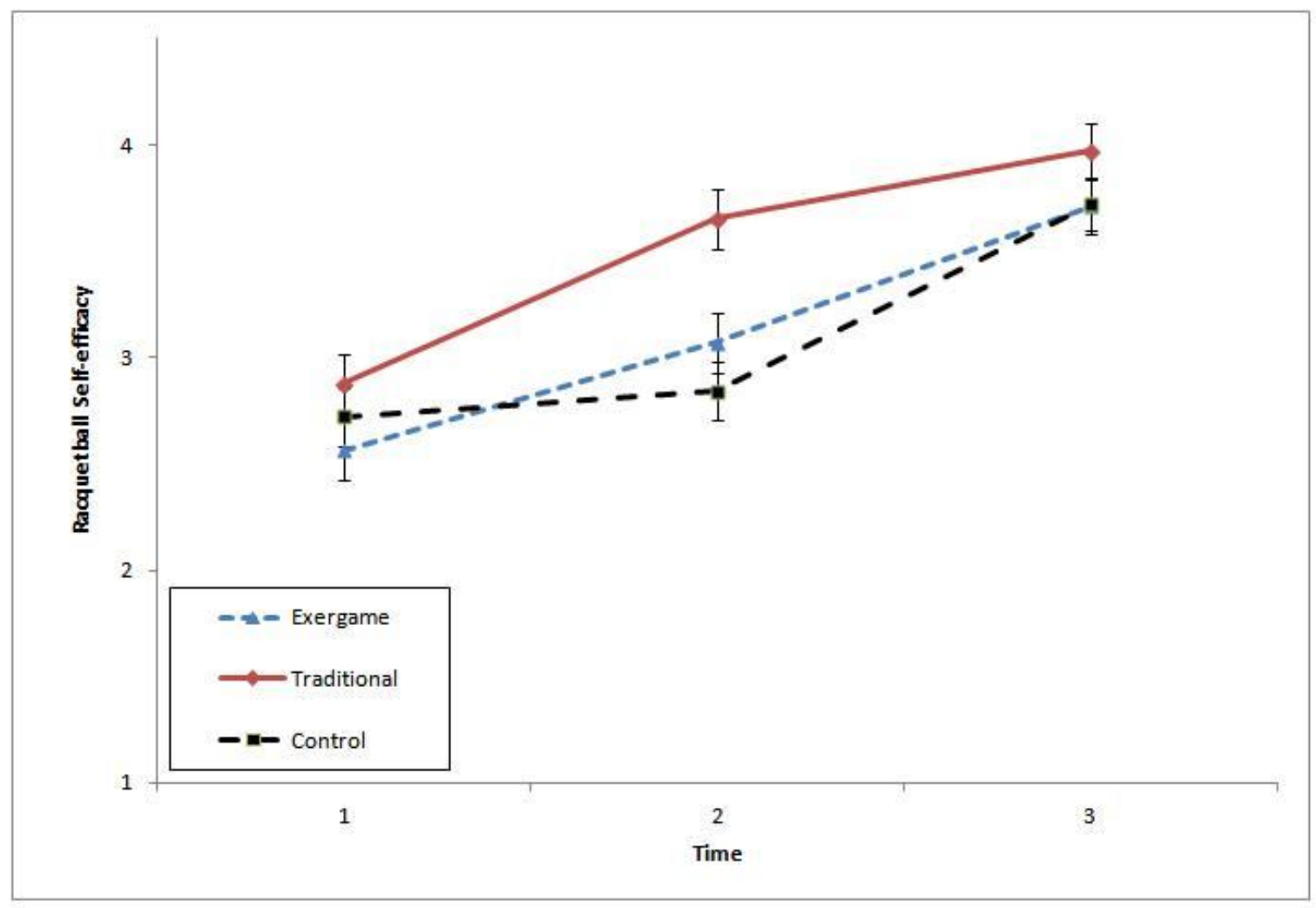

Figure 3.6 Racquetball Self-efficacy for the Significant Interaction of Time by Training Group. 


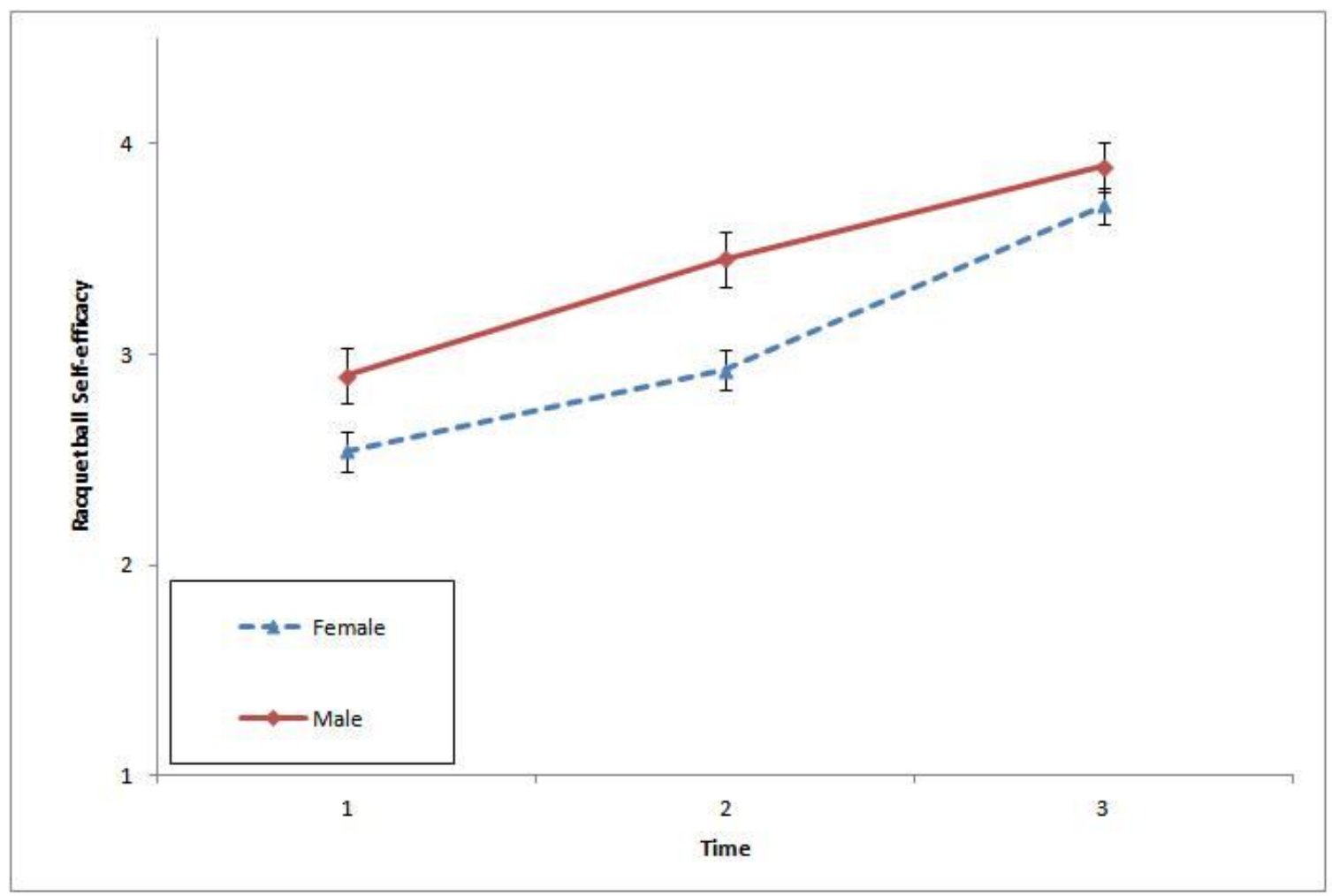

Figure 3.7 Racquetball Self-efficacy for the Significant Interaction of Time by Gender.

For the analyses with self-efficacy to regulate exercise as the outcome, only the covariate of department of participant was significant, $F(1,86)=5.49, p=.021$, partial $\eta^{2}=.06$. Participants from Kinesiology reported greater values than those from Psychology. All main effects of time, training group and gender were non-significant in this model, $\mathrm{F}(2,172)=1.60, p$ $=.205$, partial $\eta^{2}=.02, \mathrm{~F}(2,86)=.29 p=.753$, partial $\eta^{2}=.01$ and $\mathrm{F}(1,86)=.00, p=.997$, partial $\eta^{2}=.00$, respectively. The interaction effects of time by group, group by gender, time by gender and time by group by gender were also not significant, $F(4,172)=.52, p=.719$, partial $\eta^{2}=.01, F(2,86)=.45, p=.641$, partial $\eta^{2}=.01, F(2,172)=1.13, p=.325$, partial $\eta^{2}=.01$ and $\mathrm{F}(4,172)=.97, p=.425$, partial $\eta^{2}=.02$, respectively.

For the analysis of enjoyment measures for this hypothesis a significant main effect of time was discovered, $F(2,168)=8.09, p<.001$, partial $\eta^{2}=.09$. However, the main effects of 
gender and training group failed to reach significance, $F(1,84)=2.55, p=.114$, partial $\eta^{2}=.03$ and $F(2,84)=1.18, p=.314$, partial $\eta^{2}=.03$, respectively. Upon examination of pairwise comparisons for time, enjoyment scores were found to have increased with each successive time point. Inspections of interactions within the model produced significant interactions of time by group and time by group by gender, $F(4,168)=3.52, p=.009$, partial $\eta^{2}=.08$ and $F(4,168)=$ 2.53, $p=.042$, partial $\eta^{2}=.06$, respectively. However, the interactions of group by gender and time by gender were not significant, $F(2,84)=1.07, p=.348$, partial $\eta^{2}=.03$ and $F(2,168)=$ 2.57, $p=.079$, partial $\eta^{2}=.03$, respectively. Post hoc results for time by group (see Figure 3.8) showed that all groups increased enjoyment scores at every time point, with the exception of the traditional training group which only increased from T1 to T3 and the control group which exhibited higher enjoyment at T1 than T2. Additionally, the traditional training group had significantly higher enjoyment scores at T2 than did the control group. Post hoc analyses for the three-way interaction (see Figure 3.9) of time by group by gender revealed that females specifically were seen to significantly increase enjoyment at every time point, with the exception of females in the control group showing more enjoyment at T1 than at T2. Additionally, the females in the traditional training group were found to show greater enjoyment than females in all other groups at T2. 


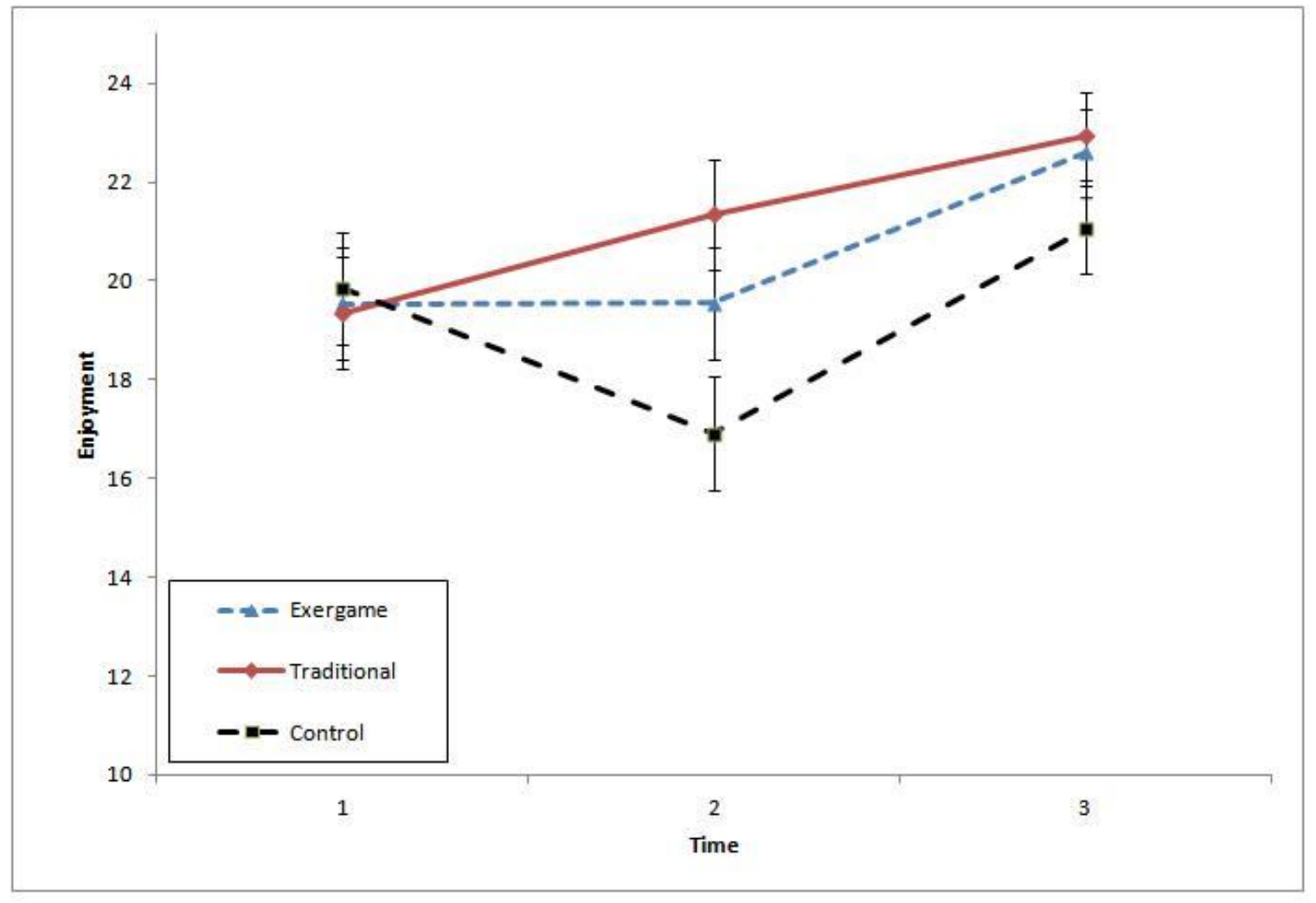

Figure 3.8 Enjoyment for the Significant Interaction of Time by Training Group. 


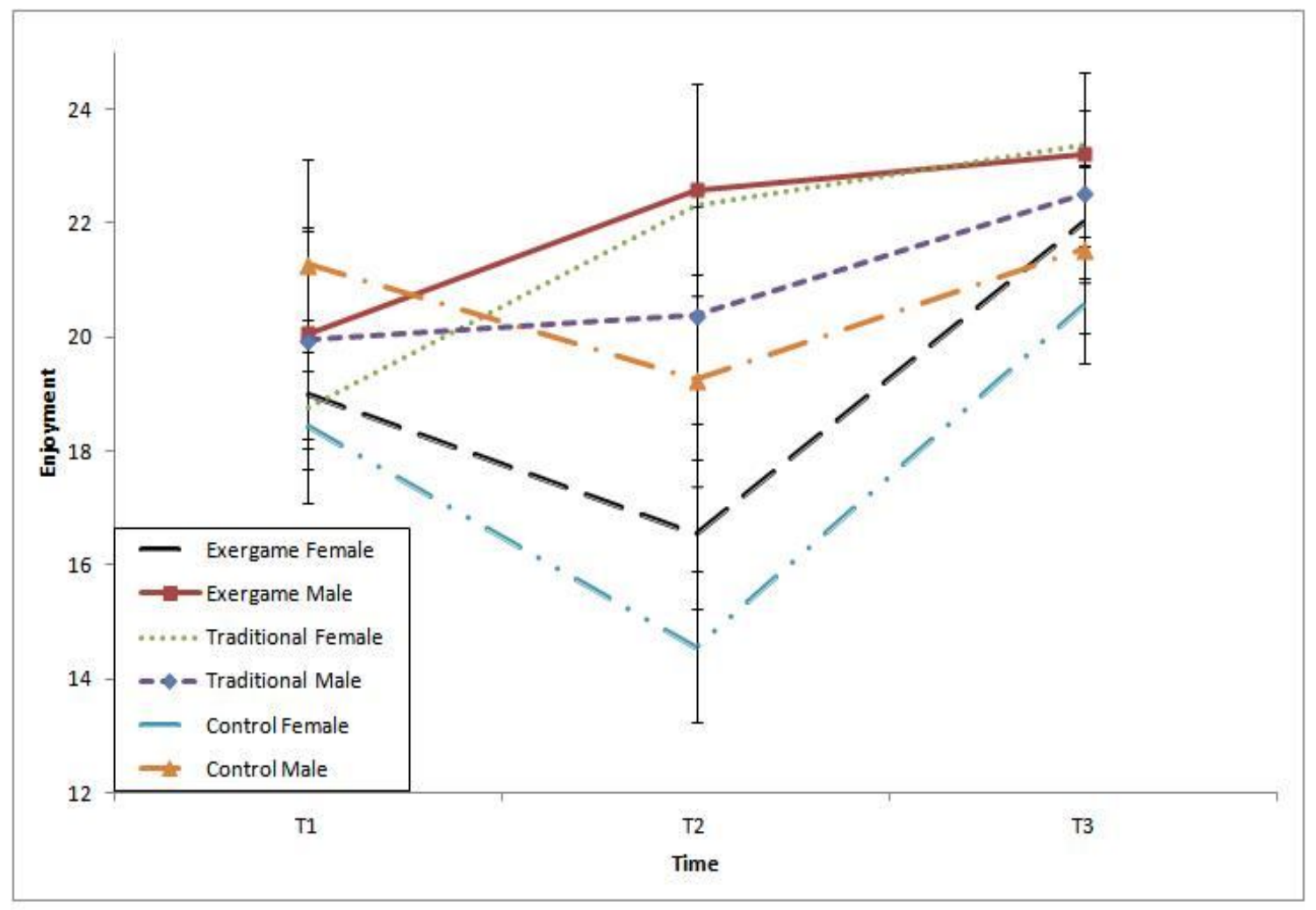

Figure 3.9 Enjoyment for the Significant Interaction of Time by Training Group by Gender.

Altogether, the analyses conducted for Hypothesis 1 discovered increases across time for all outcome variables, with the exception of exercise self-efficacy. Notably, increases for activity (as measured by accelerometer counts) were seen mostly as a function of Weeks $5-8$ being greater than Weeks 1- 4 in both the exergame and control groups. Skills test performance, activity and self-efficacy for racquetball were found to be greater in males than females. Interactions of time by training group were found to be significant for skills test performance, enjoyment, activity (as measured by accelerometer counts), and racquetball selfefficacy. Post hoc testing revealed that most of these group differences were found to be driven by the traditional training group having significantly higher values than the exergame group (racquetball self-efficacy at T2 and activity at Weeks $1-4)$ and the control group (skills test performance, enjoyment and racquetball self-efficacy at T2; activity at Weeks 1 - 4). Most 
importantly, the exergame and traditional training groups were also found to have greater skills test performance than the control group at T3. However, examination of post hocs for the threeway interaction of time by group by gender revealed that this latter effect was specific to males. Further probing of the three-way interactions found that enjoyment and activity increased across groups as a function of being female and that marked increases in activity were found for Weeks $5-8$ as compared to Weeks $1-4$ in the exergame and control groups. As such, gender effects were also found for activity in that males showed higher performance than did females for Weeks 1, 2 and 4 for the traditional training group and Weeks 6, 7 and 8 for the exergame group. Although greater enjoyment, exercise self-efficacy and activity were not seen for the exergame group above all other groups, greater skills test performance was seen for the exergame group above the control group. Furthermore, differences were not found between the exergame and traditional training group for the variables of enjoyment, skills test performance, exercise self-efficacy, and activity (Weeks $5-8$ ). Therefore, these data found partial support for Hypothesis 1.

\subsubsection{Hypothesis 2}

The second hypothesis stated that participants in the exergame condition would report greater general physical activity, greater future intention for general physical activity and greater fitness center use (MAC use) than all other groups, from the beginning to the end of the semester. Individual ANCOVA models with repeated measures were examined for each outcome except fitness center use, as this outcome was measured at one time point. See Table 3.4 for sample sizes, means, and standard errors for the outcome variables used in Hypothesis 2.

The model testing group differences across time for general physical activity (selfreport, Godin) discovered main effects of both time and training group, $F(2,162)=7.12, p=$ .001 , partial $\eta^{2}=.08$, and $F(2,81)=3.24, p=.044$, partial $\eta^{2}=.07$, respectively. Despite these main effects reaching significance, the between subjects variable of gender did not, $F(1,81)=$ 
$.58, p=.450$, partial $\eta^{2}=.01$. Pairwise comparisons for time revealed that both T2 and T3 showed higher levels of general activity than did $\mathrm{T} 1$; and that the traditional training group reported greater activity than the control group, but only at T2 (see Table 3.4). The interactions of time by training group, group by gender, time by gender, and time by group by gender all failed to reach significance and were not probed further, $F(4,162)=.33, p=.852$, partial $\eta^{2}=$ $.02, F(2,81)=.80, p=.453$, partial $\eta^{2}=.02, F(2,162)=.11, p=.899$, partial $\eta^{2}=.00$, and $F(4$, $162)=.83, p=.506$, partial $\eta^{2}=.02$, respectively. 
Table 3.4 Training Group Means and Standard Errors for Hypothesis 2 Outcomes at Week 0 - Baseline (T1), After Week 4 - Transition (T2), and After Week 8 - Completion of Training (T3).

\begin{tabular}{|c|c|c|c|c|c|c|c|c|c|c|c|c|}
\hline \multirow{2}{*}{ Variable } & \multicolumn{3}{|c|}{ Exergame } & \multicolumn{3}{|c|}{ Traditional } & \multicolumn{3}{|c|}{ Control } & \multicolumn{3}{|c|}{ Total Sample } \\
\hline & $n$ & $M$ & $(S E)$ & $n$ & $M$ & $(S E)$ & $n$ & $M$ & $(S E)$ & $n$ & & $(S E)$ \\
\hline $\begin{array}{l}\text { Activity† } \\
\text { (self-report) }\end{array}$ & 30 & & & 27 & & & 31 & & & 88 & & \\
\hline $\mathrm{T} 1$ & & 5.13 & 0.42 & & 6.53 & 0.44 & & 5.39 & 0.41 & & $5.68^{1,2}$ & 0.26 \\
\hline $\mathrm{T} 2$ & & 6.20 & 0.38 & & 7.53 & 0.40 & & 5.85 & 0.37 & & $6.53^{1}$ & 0.23 \\
\hline $\begin{array}{l}\text { T3 } \\
\text { Total }\end{array}$ & & $\begin{array}{l}6.54 \\
5.96\end{array}$ & $\begin{array}{l}0.41 \\
0.34\end{array}$ & & $\begin{array}{r}7.39 \\
7.45^{\mathrm{b}}\end{array}$ & $\begin{array}{l}0.43 \\
0.35\end{array}$ & & $\begin{array}{r}6.26 \\
5.83^{\mathrm{b}}\end{array}$ & $\begin{array}{l}0.40 \\
0.33\end{array}$ & & $6.73^{2}$ & 0.25 \\
\hline $\begin{array}{l}\text { Intention for } \\
\text { future activity }\end{array}$ & 31 & & & 31 & & & 31 & & & 93 & & \\
\hline $\mathrm{T} 1$ & & 5.21 & 0.39 & & 6.29 & 0.39 & & 5.74 & 0.38 & & $5.75^{1}$ & 0.23 \\
\hline T2 & & 5.88 & 0.33 & & 6.85 & 0.33 & & 6.50 & 0.33 & & $6.41^{1,2}$ & 0.20 \\
\hline $\begin{array}{l}\text { T3 } \\
\text { Total }\end{array}$ & & $\begin{array}{l}6.81 \\
5.97\end{array}$ & $\begin{array}{l}0.33 \\
0.27\end{array}$ & & $\begin{array}{l}7.56 \\
6.90\end{array}$ & $\begin{array}{l}0.33 \\
0.27\end{array}$ & & $\begin{array}{l}6.73 \\
6.32\end{array}$ & $\begin{array}{l}0.33 \\
0.27\end{array}$ & & $7.03^{1,2}$ & 0.20 \\
\hline $\begin{array}{l}\text { Fitness center } \\
\text { (MAC) use } \\
\text { Total }\end{array}$ & 31 & 0.99 & 0.10 & 32 & 1.02 & 0.10 & 31 & 1.08 & 0.10 & 94 & 1.03 & 0.06 \\
\hline
\end{tabular}

†Square root transformation applied to normalize distribution of activity (self-report; Godin). ${ }^{a}$ Log transformation applied to normalize distribution of fitness center use. Note: Means in the same row with the same superscript letter are significantly different. Means in the same column within the same group that have the same superscript number are significantly different. 
Future intention for physical activity was subsequently tested and was found to violate the assumption of sphericity. Thus, the Greenhouse-Geisser test was examined for this analysis as a correction for this violation. The results revealed a main effect of time, $F(1.81,155.84)=$ $3.21, p=.043$, partial $\eta^{2}=.04$. However, the main effects of training group and gender were not found to be significant, $\mathrm{F}(2,86)=2.15, p=.123$, partial $\eta^{2}=.05$, and $F(1,86)=.09, p=.764$, partial $\eta^{2}=.00$. Post hoc testing of the significant main effect showed that greater reports of intentions for future physical activity were reported at each successive time point (see Table 3.4). Interaction effects of time by group, group by gender, time by gender and time by group by gender were not found to be significant, $F(3.62,155.84)=.49, p=.743$, partial $\eta^{2}=.01, F(2$, $86)=.34, p=.710$, partial $\eta^{2}=.01, F(1.81,155.84)=.20, p=.801$, partial $\eta^{2}=.00$ and $F(3.62$, $155.84)=.66, p=.608$, partial $\eta^{2}=.02$, respectively.

The ANCOVA model (without repeated measures) conducted for the variable of fitness center (MAC) use revealed only a main effect of gender and a main effect of the covariate participant department, $F(1,87)=10.71, p=.002$, partial $\eta^{2}=.11$ and $F(1,87)=8.15, p=.005$, partial $\eta^{2}=.09$, respectively. The main effect of training group and the interaction of group by gender failed to reach significance, $\mathrm{F}(2,87)=.45, p=.640$, partial $\eta^{2}=.01 \& \mathrm{~F}(2,87)=.06, p=$ .940 , partial $\eta^{2}=.00$, respectively. Upon inspection of pairwise comparisons for gender, it was found that males $(M=1.23, S E=.10)$ reported greater use of the university fitness center than did females $(M=.82, S E=.07)$. Post hoc comparisons also revealed that those participants from the Kinesiology Department $(M=26.68, S E=3.15)$ used the fitness center more than those in Psychology $(M=14.86, S E=2.72)$.

Overall, statistical models conducted for Hypothesis 2 revealed increases in the dependent variables of general physical activity and intention for future physical activity across time for all groups. As such, it was discovered that the traditional training group reported higher general physical activity than the control group at T2. Despite this, no group differences were discovered between the exergame and traditional training groups for any of the outcome 
variables used in Hypothesis 2. Moreover, gender and department of participant were found to predict fitness center use, while no group by time interactions or three-way interactions of time by group by gender were discovered for any of these analyses. Therefore, these data did not provide support for Hypothesis 2.

\subsubsection{Hypothesis 3}

The third prediction, which stated that enjoyment and self-efficacy would mediate the relationship between condition and the outcome measures of skills test performance, activity (as measured by an accelerometer), future intention for physical activity, activity center use, and actual physical activity across all time points, was planned to be analyzed using a hierarchical multiple regression analysis with the Preacher and Hayes method to test mediation (Preacher, \& Hayes, 2004). However, this hypothesis was contingent upon discovering group differences among the exergame, traditional training, and control groups while testing previous hypotheses and through additional statistical probing. As the predicted significant training group relationships were not found, these analyses were not able to be conducted. Thus, the proposed mediational relationships did not exist in these data, and Hypothesis 3 was not supported.

\subsubsection{Feedback}

Participant feedback was collected at T3 for all participants using a variety of questions asking for comments and participant views on study satisfaction, instruction satisfaction, whether or not an exercise routine was started, how influential the study was to exercise habits, and how enjoyable the study was overall (see Table 3.5). Examination of frequencies and descriptive statistics found that the majority of participants were very satisfied with both the study overall and with the instruction they were given. The majority of participants also reported the study influenced their adoption of a new exercise routine, a regular exercise routine, and that the study was very influential in the adoption of these routines. The vast majority of participants also reported that they would not change anything with the study. Furthermore, a 
total of seven comments were left concerning desired changes to the study. These comments consisted of study design change suggestions, the desire for longer training sessions, and the wish for further instruction. Comments not regarding changes were overwhelmingly positive, praising the enjoyment level of the study, the positive influence on exercise habits, the quality of the instruction, and many comments praising the sport itself.

Table 3.5 Descriptive Statistics for Study Feedback Variables.

\begin{tabular}{|c|c|c|c|c|}
\hline Variable & & $N$ & Mean & $\begin{array}{l}\text { Standard } \\
\text { Deviation }\end{array}$ \\
\hline Overall satisfaction & & 93 & 4.77 & 0.47 \\
\hline Instruction satisfaction & & 94 & 4.82 & 0.49 \\
\hline Influence on exercise habits & & 94 & 3.72 & 1.17 \\
\hline Activities were enjoyable & & 93 & 4.82 & 0.44 \\
\hline Influence, new adoption & & 79 & 3.65 & 1.21 \\
\hline Influence, began exercise & & 83 & 3.51 & 1.35 \\
\hline \multirow[t]{2}{*}{ Influence, adopt regular } & & 82 & 3.45 & 1.41 \\
\hline & & Frequency & Percent & \\
\hline \multirow[t]{3}{*}{ Change Study } & & 93 & - & \\
\hline & No & 85 & $82.5 \%$ & \\
\hline & Yes & 8 & $7.8 \%$ & \\
\hline \multirow[t]{3}{*}{ Adopted new exercise routine? } & & 93 & - & \\
\hline & No & 14 & $15 \%$ & \\
\hline & Yes & 79 & $85 \%$ & \\
\hline \multirow[t]{3}{*}{ Began exercising during study Semester? } & & 93 & - & \\
\hline & No & 10 & $10.8 \%$ & \\
\hline & Yes & 83 & $89.2 \%$ & \\
\hline \multirow[t]{3}{*}{ Began regular exercise routine? } & & 94 & - & \\
\hline & No & 12 & $12.8 \%$ & \\
\hline & Yes & 82 & $87.2 \%$ & \\
\hline
\end{tabular}




\section{CHAPTER 4 \\ DISCUSSION}

This study sought to provide evidence that exergame use could be a viable gateway to a particular physical activity by showing that the exergame group generated greater levels of the physical and cognitive physical activity measures than did the control group, supporting the design's efficacy as an intervention. Similarly, the exergame group was expected to also experience greater than or equal scores for these measures when compared to the traditional training group; supporting exergame use as either a superior or equivalent training method compared to the traditional training method. However, sufficient evidence to support this design as an effective intervention or as a comparable training method to the traditional method was not discovered from these data.

Hypothesis 1 was given partial support in that the exergame group was found to have greater skills test performance than the control group at T3 (with males) and that no differences were found between the exergame and traditional training groups for the variables of enjoyment, skills test performance, exercise self-efficacy, and activity (Weeks 5 - 8). However, greater enjoyment, exercise self-efficacy and activity were not seen for the exergame group above all other groups. Furthermore, analyses run for Hypothesis 1 discovered increases across time for all outcome variables, with the exception of exercise self-efficacy. Additionally, increases for activity (as measured by accelerometer counts) were seen mostly in females, activity for Weeks $5-8$ were greater than Weeks 1 - 4 in both the exergame and control groups and skills test performance, activity and self-efficacy for racquetball were found to be greater in males than females. Lastly, most group differences for the variables of skills test performance, enjoyment, activity (as measured by accelerometer counts), and racquetball self-efficacy were discovered to be the traditional training group above the control group (skills test performance, 
enjoyment and racquetball self-efficacy at T2; activity at weeks $1-4)$, as well as the exergame group (racquetball self-efficacy at T2 and activity at Weeks 1 - 4).

Upon examining analysis results, no support for Hypothesis 2 was found in these data. Although statistical models conducted for Hypothesis 2 revealed increases in the dependent variables of general physical activity and intention for future physical activity across time for all groups, the traditional training group having higher general physical activity than the control group was the only group difference discovered. Thus, the exergame group was not found to have higher levels of the outcome variables than any other group. Additionally, no group differences were found between the exergame group and the traditional training group for any outcome. Moreover, gender and department of participant were found to predict fitness center use, but no group by time interactions or three-way interactions were discovered for any of these analyses. Hypothesis 3 predicted that enjoyment and self-efficacy would mediate the relationship between condition and the outcome measures of skills test performance, activity (as measured by an accelerometer), future intention for physical activity, activity center use, and actual physical activity across all time points. However, this hypothesis was dependent upon finding the expected array of group differences entailed in Hypotheses 1 and 2. Results of the analyses testing these hypotheses did not find the expected group relationships. Therefore, the proposed analyses for the mediational relationships in Hypothesis 3 were unable to be conducted and these relationships did not exist in these data.

Significant effects of time and group were discovered. Marked increases were seen across time in the majority of the physical and cognitive measures of physical activity used for the purposes of this study. For the majority of analyses, the traditional training group surfaced as the group exhibiting significant increases and greater levels of activity, skills test performance, racquetball self-efficacy, and enjoyment at T2 above other groups. However, notable differences between the exergame, traditional training and control groups at T3 for skills tests performance supported expectations that exergame training would be higher than control 
methods and greater than or equal to traditional training methods. The differences between exergame and control groups were likely due to the differences in introductory training. Although activity levels were not different between these two groups, playing exergames gives the player simulated experience with the rules, techniques, and basic behaviors involved in the sport involved. The effects of these physical and cognitive aspects were demonstrated in previous research, where the confidence of exergame players was increased due to playtime (Murphy, et al., 2009). However, the discovery that the exergame group did not perform better than the traditional training group may speak to the conclusions previous researchers have come to regarding exergames being used as a supplementary or introductory medium of physical activity, and not as a replacement for traditional activity (Lanningham-Foster, 2009). Those participants in the traditional training group received eight weeks of first-person training for their sport. This would be difficult to compare as a video game simulation does not involve all the activities that the actual sport does.

Furthermore, it was discovered that no group differences existed between exergame and traditional training groups on the outcome variables of enjoyment, skills test performance, exercise self-efficacy, activity (Weeks $5-8$ ), general physical activity or future intention. These findings may be an effect of the particular population sampled for this research. Past exergame research has primarily focused on testing exergame use with elderly adult populations (Jarvis, 2010). Studies have reported increases in enjoyment, balance, mobility, functionality, and willingness to participate in physical activity. However, these particular benefits may not apply in the same manner to a younger, more able-bodied population, such as undergraduate college students. These individuals are likely to have no problems with functionality, balance, or mobility. Therefore, the reward of greater benefit attainment that coincides with exergame use in the elderly may be lessened with young adults, and may lead to dampening of the relationship between exergame use and increases in physical and cognitive physical activity outcomes. Additionally, levels of enjoyment, racquetball self-efficacy, and adherence as a whole for the 
study were very high. This may have resulted in any possible group differences being undetectable in analyses.

Analytical results also showed that enjoyment scores and activity data were reduced in concordance with the aims of the control methods and manipulations, such that enjoyment was greater at T1 than T2 and activity accelerometer counts were greater during Weeks $5-8$ than during Weeks $1-4$. These findings, as a whole, when considered alongside the low degree of improvement on the outcome variables from T1 to T2 by the control group, provide support that the study design was effective for its intended control measures and manipulations. Control measures included selecting a sample that had little or no experience with the specific sport, selecting for those who were not regular exercisers, ensuring training groups only played with members within their own group, providing the same specific sport training to all groups at the same time point and not revealing the specific sport selected until the end of the first assessment.

Concurrently, the study design included the use of three groups so that those in the exergame condition could objectively be compared to those who had no prior training as a control, as well as those who train in the traditional method for an additional comparison to typical methods. Additionally, the design involved transitioning both exergame and control groups not only as a direct comparison for the identification of a gateway mechanism, but also for the measurement of the aftereffects of introductory exergame training. As the current project was designed to explore the possibility of a gateway mechanism, the control and manipulations inherent to the design were effective in their purpose. The effectiveness of the transition into playing the actual sport was supported in that activity levels increased significantly after the transition took place, as well as due to the continued increase in enjoyment found at T3. Enjoyment has been suggested to be a major contributing factor to the effectiveness of exergames (Sell, Lillie, \& Taylor, 2008). As such, the results simultaneously showing greater enjoyment at T3 for both exergame and control groups, and yet reduced enjoyment from T1 to 
T2 for the control group suggests that this control measure was indeed effective. Research has reported the benefits of enjoyment on sport participation in that those with higher enjoyment tend to be more committed to the sport (Hagberg et al., 2009). This evidence is also further supported when considering the attrition of the current project was a mere $9 \%$ whereas the typical attrition rates for exercise interventions with behavioral components fall in between $18 \%$ and 34\% (Linke, 2011).

Other strengths of the current study involved the randomization procedures to ensure equal representations of participants in all training groups. Player preference, demographic background and previous experience with video games can be confounding factors when designing a study around exergames. Previous research with exergames has neglected to control for such factors (Murphy et al., 2009 \& Sell, Lillie, \& Taylor, 2008). However, this study used randomization, prescreening questionnaires, and specific eligibility criteria to control for these confounding variables.

Previous research on exergame interventions reported marked increases in the willingness of adolescents to participate in future physical activity (Murphy et al., 2009 \& Kepka, 2011). These increases in willingness to perform physical activity provided early evidence that while exergame use was not a sufficient exercise medium in itself, it may have served as a gateway or introductory medium towards more intense activities. However, these reports of willingness did not specifically differentiate whether the willingness occurred for general physical activity, specific activity, or for one over the other. Accordingly, the current project was designed to include measures which assessed both possibilities. At face value the measure of self-reported willingness was only gauging the future intention of the adolescent to take part in physical activities. Thus, it was left undetermined whether these intentions were translated into actual behaviors. As such, the current study included measures of general and sport specific physical activity, intention for physical activity, as well as multiple measures of self-report and 
actual physical activity. Although analysis of these measures did not find increased intention or physical activity with the exergame group, the results did uncover other influential factors.

Despite not finding full support for expectations set forth by previous research, the findings uncovered other crucial relationships. Although previous literature provided preliminary evidence that a gateway mechanism may exist, this possibility was not fully supported in the current study (Murphy, et al., 2007 \& Kepka, 2011). Results from this study indicated improvement in the exergame group for racquetball self-efficacy, activity, enjoyment of the sport and skills tests performance; all measures that were specific to the sport played. Concurrently, there were not improvements in general physical activity levels, fitness center use, or selfefficacy for general physical activity in the exergame group; these being measures relating to general physical activity. These findings suggested that the gateway mechanism, or at least exergame use with the intention of promoting exercise, may be most efficacious for specific activity rather than for general physical activity.

Other influential relationships discovered in this research included the observed influence that gender may play on the exergame - physical activity relationship. Findings indicating that gender was a significant predictor of skills test performance, racquetball selfefficacy, activity, and fitness center use also provided important information suggesting that males and females may respond to exergame play and actual sport play in different ways. Past research into gender differences and physical activity suggested that differences could occur through either biological differences, cognitive differences, or both (De Moor, et al., 2007, Edwards, Bocarro, Kanters, \& Casper, 2011, \& Lam, Sit, \& McManus, 2011). Moreover, Morgan (2001) emphasized the importance of purposeful activity. Morgan reports that adherence rates increase with the implementation of activities that have more purpose, such as walking for transportation purposes as opposed to walking on a treadmill. Furthermore, a participant's preferred exertion (how intense one prefers to exercise) while participating in these activities was suggested to be another influential factor that is often different between genders (Morgan, 
2001). As the sport of racquetball is a purposeful activity, the activity-related gender differences found for the current study may be a function of differences due to preferred exertion. Additionally, the low attrition rate $(9 \%)$ found within the current study may also be a product of the specific activity being a purposeful activity.

As the notion that exergame use may lead to further physical activity is rather new, few studies have explored this area directly or indirectly (Murphy et al., 2009 \& Kepka, 2011). However, these select few, when compared to the current study, involved larger sample sizes, longer overall training periods, and different samples. As such, results from the current study indicating marginally significant increased outcome values for the exergame group above the control group may have also indicated that more time, more participants, or different samples may have been needed to illuminate these effects. Furthermore, Murphy and colleagues in their study of exergame effects used the exergame called "Dance Dance Revolution" (DDR) as the only game provided to participants. Although DDR is similar to a number of other dance exergames on the market, when considering exergames as a whole this particular type of game is atypical. DDR has been suggested to actually induce moderate and even strenuous activity out of the player (Murphy et al., 2009), whereas the vast majority of all exergames have only been found to induce low intensity activity (Miyachi, et al., 2010). The findings from the current study were consistent with this research as the majority of exergame training sessions inducing only low levels of activity (Miyachi, et al., 2010). Additionally, other exergame research has successfully used accelerometers for the measurement of activity during exergame play without voicing the concern of limited motion due to game play parameters (Lanningham-Foster et al., 2009). Although it was not logistically possible in the current study, future research in this area may benefit from the addition of heart rate monitors for the measurement of activity through a medium that is less constrained with the need for directionalized movement.

Although the findings of this study only partially supported the notion of a gateway mechanism for exergame use, a number of additional relationships and influential factors were 
discovered from these findings, including gender differences and specific versus general physical activity differences. Overall findings, however, did produce results that supported future exploration in this area. Notably, greater skills test performance over and above the control group, as well as differences not being seen between exergame and traditional training groups for the majority of outcome variables provides support for the use of introductory exergame training as a suitable means of promoting physical activity. These results indicated that greater racquetball skill in male participants was attained through exergame use and that beginning a sport through an exergame was likely a comparable method to training in the traditional fashion. This prospect was also supported by outside research. The use of low intensity activity for the introductory period of an exercise routine was recommended by the American College of Sports Medicine and has been associated with increased adherence and reduced injury (Haskell et al., 2007). Thus, the low intensity activity required of exergame use may facilitate its effects on adoption of regular physical activity, with an even greater effect likely if the exergame is specific to the sport in which one is transitioning.

\section{$\underline{4.1 \text { Limitations }}$}

Although previous literature has supported the notion that exergame use might serve as a gateway to a specific or general physical activity (Murphy et al., 2009 \& Kepka, 2011), this mechanism was only partially supported within these particular analyses. While it was expected that more outcomes would be elevated among those in the exergame group as a function of increased enjoyment, many of these outcomes may have been muted due to the nature of the exergame platform itself. As a major physiological outcome, activity (as measured by accelerometer counts) was likely hindered by the exergame player's inability to move out of the video game sensor's field of view without causing a loss of connection from the hand-held remote controller. This limited movement was also compounded by the game itself, which consumed somewhere between 3 and 6 minutes of the participants' training sessions in order to navigate through options menus, set up games, and calibrate remotes to the platform device. 
Additionally, it was voiced by some participants after transition from exergame to actual racquetball that the exergame was not a good proxy for the sport, as slight changes were made to the rules of the exergame to make it feasible as a video game. Due to these limitations, other variables may have also been influenced by these factors. Self-efficacy for racquetball was likely affected in this respect, as the aspects that commonly change self-efficacy are education and experience. Results indicating a greater level of racquetball self-efficacy for the traditional training group above the exergame group at T2 support this possibility.

Most relevant to the results of this study, there appeared to be an unexpected ceiling effect for the measurement of enjoyment of racquetball in each group. The majority of participants indicated that they enjoyed their group activities with mean scores in the exergame and traditional training groups averaging over time between 4.4 and 4.6 on a Likert scale from 1 to 5 . This event was not anticipated by the researchers as this scale was extensively prevalidated and had not been found to have produced this effect previously. While it is definitely a strength of the project that the training groups enjoyed the sport, there was little variability among the groups and differences could not be detected. Future research should consider using multiple indicators of satisfaction and enjoyment.

\subsection{Future Directions and Conclusions}

The results of the current study advance the field on exergame research, provide partial support for the novel use of exergames as a gateway mechanism to physical activity, and may provide future guidelines by which to design exergame programs. Although expectations were not fully supported, these data demonstrate that increases in skills test performance, enjoyment, activity, intentions for future physical activity, and racquetball self-efficacy were seen across time with introductory exergame training including those individuals in the exergame group. While these increases may not have had the power to rise over and above the levels of the other groups for most cases, these findings suggest that individuals of varying genders and backgrounds with exercise experience respond differently to exergame use as a means of 
influencing physical activity. Moreover, the type of exergame, the duration of time exposed to the exergame, and whether or not training is for a specific activity may also be influential factors as for determining the efficacy or existence of a possible gateway mechanism.

Future research should build on these findings in a number of ways. First, gender appears to be a major influential factor. Exergame interventions may need to be tailored for the needs and preferences of each gender. Additionally, some exergames (e.g., Wii Fit) are already marketed to specific genders in the retail marketplace and that may affect their adoption and use for physical activity. Second, future research needs to explore the possibility that the gateway mechanism may be better used for specific activities as opposed to general physical activities. It will be important to determine if any effects from exergame use can be generalized to other forms of physical activity by examining individuals over longer periods of time. Third, this was the first study to directly compare sport-specific exergame use to the actual sport for both physical and cognitive physical activity measures, and more research needs to be done comparing other sports. The findings may vary depending on what type of sport is the focus. Future research addressing these points may produce further support for the possibility of using the flood of exergames entering the entertainment market as an enjoyable, feasible and accessible method of addressing the alarming need for regular physical activity among Americans. 


\section{REFERENCES}

Adnan, Y., McKenzie, A. \& Miyahara, M. (2001). Self-efficacy for quad rugby skills and activities of daily living. Adapted Physical Activity Quarterly, 18, 90-101.

Ajzen, I. (1985). From intentions to action: A theory of planned behavior. In J. Kuhl \& J. Beckman (Eds.), Action control: From cognitions to behaviors (pp. 11-39). New York, NY: Springer.

Bandura, A. (2006). Guide for Constructing Self-efficacy Scales. In T. Urdan 7 F. Pajares (Eds) Self-Efficacy Beliefs of Adolescents, 307-337.

Bandura, A. (1998). Health promotion from the perspective of social cognitive theory. Psychology and Health, 13, 623-649.

Casper, J. M., \& Stellino, M. (2008). Demographic Predictors of Recreational Tennis Participants' Sport Commitment. Journal of Park \& Recreation Administration, 26(3), 95115.

Center for Disease Control (2010). U.S. Physical Activity Statistics. Retreived from http://apps.nccd.cdc.gov/PASurveillance/StateSumResultV.asp

Courneya, K., \& Bobick, T. (2000). Integrating the theory of planned behavior with the processes and stages of change in the exercise domain. Psychology of Sport \& Exercise, 1(1), 41- 56.

De Moor, M., Posthuma, D., Hottenga, J., Willemsen, G., Boomsma, D., \& De Geus, E. (2007). Genome-wide Linkage Scan for Exercise Participation in Dutch Sibling Pairs. European Journal of Human Genetics, 15(12), 1252-1259. doi: 10.1038/sj.ejhg.5201907. Dishman, R. K. (1994). Predicting and changing exercise and physical activity: What's practical and what's not. In H. Quinn, L. Gauvin, \& A.Wall (Eds.), Human Kinetics (pp. 97-106). Champaign, IL: Toward Active Living. 
Dougall, A. L., Swanson, J. N., Grimm, J. R., Jenney, C. T., \& Frame, M. C. (2011). Tempering the decline in college student physical activity using informational interventions: Moderating effects of stress and stage of change. Journal of Applied Biobehavioral Research, 16(1), 16-41. doi: 10.1111/j.1751-9861.2011.00064.x

Edwards, M. B., Bocarro, J. N., Kanters, M., \& Casper, J. (2011). Participation in Interscholastic and Intramural Sport Programs in Middle Schools: An Exploratory Investigation of Race and Gender. Recreational Sports Journal, 35(2), 157-173.

Godin, F., Shephard, R. J.. (1997) Godin Leisure-Time Exercise Questionnaire. Medicine and Sciene in Sports and Exercise. 29 June Supplement: S36-S38.

Grubbs, L., \& Carter, J. (2002). The Relationship of Perceived Benefits and Barriers to Reported Exercise Behaviors in College Undergraduates. Fam Community Health, 25(2), 76-84.

Hagberg, L. A., Lindahl, B. B., Nyberg, L. L., \& Hellénius, M. L. (2009). Importance of enjoyment when promoting physical exercise. Scandinavian Journal of Medicine \& Science in Sports, 19(5), 740-747.

Hagger, M, Chatzisarantis, N, \& Biddle, S. (2002). A meta-analytic review of the theories of reasoned action and planned behavior in physical activity: Predictive validity and the contribution of additional variables. Journal of Sport and Exercise Psychology, 24. 3-32.

Haskell, W. L., Lee, I., Pate, R. R., Powell, K. E., Blair, S. N., Franklin, B. A., Macera, C. A., Heath, G. W., Thompson, P. D., \& Bauman, A. (2007). Physical Activity and Public Health: Updated Recommendation for Adults from the American College of Sports Medicine and the American Heart Association. Medicine \& Science in Sports \& Exercise, 39(8), 1423-1434.

Hausenblas, H., Carron, A., \& Mack, D. (1997). Application of the theories of reasoned action and planned behavior to exercise behavior: a meta-analysis. Journal of Sport and Exercise Psychology, 19. 36-51. 
Hill, C., Abraham, C., \& Wright, D. (2007). Can theory-based messages in combination with cognitive prompts promote exercise in classroom settings? Social Science and Medicine, 65, 1049-1058.

Jarvis, J. (2010, July 4). University of texas at arlington study looks at preventing falls in older adults. Star-telegram. Retrieved from http://www.star-telegram.com/2010/07/04/231345 8/University-of-texas-at-arlington.html\#ixzz0t1DDxp4h

Kepka, M. (2011). DDR video game shown to stop weight gain, improve health. San Francisco Chronicle. Retrieved from http://blog.sfgate.com/techchron/2007/02/01/ddr-video-gameshown-to-stop-weight-gain-improve-health/

Lanningham-Foster, L., Foster, R., McCrady, S., Jensen, T., Mitre, N., \& Levine, J. (2009). Activity-promoting video games and increased energy expenditure. The Journal of Pediatrics, 154. 819-823.

Lam, J., Sit, C., \& McManus, A. M. (2011). Play Pattern of Seated Video Game and Active "Exergame" Alternatives. Journal of Adolescent Health, 49(2), 24-30.

Lam, T., Ho, S., Hedley, A., Mak, K., \& Leung, G. (2004). Leisure time physical activity and mortality in Hong Kong: case-control study of all adult deaths in 1998. Annals Of Epidemiology, 14(6), 391-398.

Lam, E. \& Zhang, J. (2002). The development and validation of a racquetball skills test battery for young adult beginners. Measurement in Physical Activity Education and Exercise Science, 6, 95-126.

Linke, S. E. (2011). Attrition and adherence rates of sustained vs. intermittent exercise interventions. Annals of Behavior Medicine, 42(2), 197 - 209.

Marcus, B., Banspach, S., Lefebvre, R., Rossi, J., Carleton, R., \& Abrams, D.(1992) Using the stages of change model to increase the adoption of physical activity among community participants. American Journal of Health Promotion, 6(6), 424- 429. 
Morgan, W. P. (2001). Prescription of Physical Activity: A Paradigm Shift. Quest (00336297), 53(3), 366-398.

Miyachi , M., Yamamoto, K., Ohkawara, K., \& Tanaka, S. (2010). METs in adults while playing active video games: a metabolic chamber study. Medicine \& Science in Sports \& Exercise, 10, 1149- 1153.

Murphy, E., Carson, L., Neal, W., Baylis, E., Donley, D., \& Yeater, R. (2009). Effects of an exercise intervention using Dance Dance Revolution on endothelial function and other risk factors in overweight children. International Journal of Pediatric Obesity, 4(4) 205214.

Norman, P., \& Conner, M. (2005). The theory of planned behavior and exercise: Evidence for the mediating and moderating roles of planning on intention-behavior relationships. Journal of Sport and Exercise Psychology, 27. 488-504.

Physical Activity and Health: A Report of the Surgeon General. Atlanta, Ga: U.S. Department of Health and Human Services, Centers for Disease Control and Prevention, National Center for Chronic Disease Prevention and Health Promotion, 1996. S/N 017-02300196-5.

Preacher, K. \& Hayes, A. (2004). SPSS and SAS procedures for estimating indirect effects in simple mediation models. Behavior Research Methods, Instruments, \& Computers, 36. 717-731.

Prochaska, J. O., DiClemente, C. C., \& Norcross, J. C. (1992). In search of how people change. Applications to addictive behaviors. American Psychologist, 47(9), 1102-1114.

Safrit, M. (1990). Introduction to Measurement in Physical Education and Exercise Science. St. Louis, MO: Times Mirror/ Masby College Publishing.

Scanlan, T. K., Carpenter, P. J., Schmidt, G. W., Simons, J. P., \& Keeler, B. (1993). An introduction to the sport commitment model. Journal of Sport \& Exercise Psychology, 15, 1-15. 
Sell, K., Lillie, T., \& Taylor, J. (2008). Energy expenditure during physically interactive video game playing in male college students with different playing experience. Journal of American College Health, 56, 505-511.

Stone, E., McKenzie, T., Welk, G., \& Booth, M. (1998). Effects of physical activity interventions in youth. Review and synthesis. American Journal Of Preventive Medicine, 15(4), 298315. Retrieved from EBSCOhost.

Wallace, L., Buckworth, J., Kirby, T., \& Sherman, M. (2000). Characteristics of exercise behavior among college students: application of social cognitive theory to predicting stage of change. Preventive Medicine, 31. 494-505. 


\section{BIOGRAPHICAL INFORMATION}

Colin Timothy Jenney received his Bachelor of Science degree in Interdisciplinary Studies with a medical science focus from the University Of Texas Arlington in 2010. As an undergraduate, he began conducting applied behavioral research with the lab of Dr. Liegey Dougall. During graduate training, Colin's research has focused on the assessment and creation of novel interventions for the support of self-care behaviors in vulnerable populations, such as exercise promotion among college students and depression/stress management among Type 2 diabetics. He has certifications in personal training, phlebotomy, first aid, CPR/AED, healthy lifestyles promotion, and emergency oxygen administration. Colin seeks to continue his graduate education at the University of Texas Arlington and earn his PhD in Health Psychology and Neuroscience in 2014. 